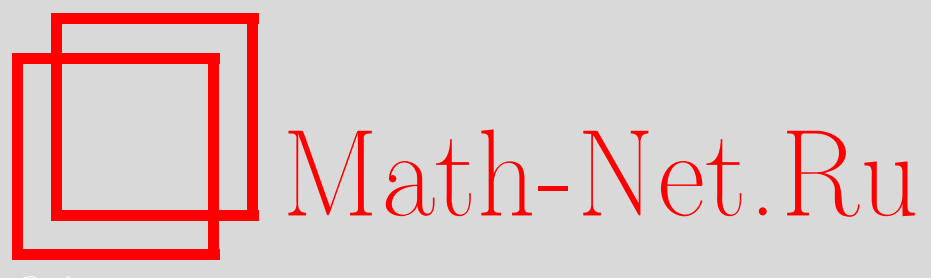

А. Г. Баскаков, Оценки функции Грина и параметров экспоненциальной дихотомии гиперболической полугруппы операторов и линейных отношений, Матем. сб., 2015, том 206, номер 8, 23-62

DOI: https://doi.org/10.4213/sm8193

Использование Общероссийского математического портала Math-Net.Ru подразумевает, что вы прочитали и согласны с пользовательским соглашением http://www . mathnet.ru/rus/agreement

Параметры загрузки:

IP: 3.95 .254 .165

26 апреля 2023 г., 13:55:29 


\title{
А. Г. Баскаков \\ Оценки функции Грина и параметров экспоненциальной дихотомии гиперболической полугруппы операторов и линейных отношений
}

\begin{abstract}
На основе применения уравнения Ляпунова, метода подобных операторов и методов гармонического анализа получены оценки параметров экспоненциальной дихотомии и функции Грина, построенной по гиперболической полугруппе операторов и гиперболическому линейному отношению. Оценки получены с использованием величин, которые определяются резольвентой инфинитезимального оператора полугруппы операторов и линейного отношения

Библиография: 51 название.
\end{abstract}

Ключевые слова: гиперболические полугруппы операторов, линейные отношения, метод подобных операторов, уравнение Ляпунова, спектр оператора.

DOI: $10.4213 / \mathrm{sm} 8193$

\section{§1. Введение. Основные понятия и результаты}

Пусть $\mathscr{H}$ - комплексное гильбертово пространство, End $\mathscr{H}$ - банахова алгебра линейных ограниченных операторов, действующих в $\mathscr{H}$. Рассматривается сильно непрерывная полугруппа операторов (полугруппа класса $C_{0}$ )

$$
T: \mathbb{R}_{+}=[0, \infty) \rightarrow \text { End } \mathscr{H},
$$

и пусть $A: D(A) \subset \mathscr{H} \rightarrow \mathscr{H}$ - ее инфинитезимальный оператор (см. [1]).

Рассмотрим линейное дифференциальное уравнение

$$
\dot{x}(t)-A x(t)=f(t), \quad t \in \mathbb{R},
$$

где функция $f: \mathbb{R} \rightarrow \mathscr{H}$ принадлежит одному из следующих банаховых пространств функций. Через $C_{b}=C_{b}(\mathbb{R}, \mathscr{H})$ обозначим банахово пространство ограниченных непрерывных функций, определенных на $\mathbb{R}$ со значениями в $\mathscr{H}$ с нормой $\|x\|_{\infty}=\sup _{t \in \mathbb{R}}\|x(t)\|$. Символ $L^{p}=L^{p}(\mathbb{R}, \mathscr{H}), p \in[1, \infty]$, используется для обозначения банахова пространства (классов) измеримых по Бохнеру функций $x: \mathbb{R} \rightarrow \mathscr{H}$, для которых конечна величина

$$
\|x\|_{p}=\left(\int_{\mathbb{R}}\|x(t)\|^{p} d t\right)^{1 / p}, \quad p \neq \infty, \quad\|x\|_{\infty}=\underset{t \in \mathbb{R}}{\operatorname{vraisup}}\|x(t)\|, \quad p=\infty .
$$

Исследование выполнено за счет гранта Российского научного фонда (проект № 14-21-00066). 
Если $p=2$, то $L^{2}(\mathbb{R}, \mathscr{H})$ является гильбертовым пространством со скалярным произведением $\langle x, y\rangle=\int_{\mathbb{R}}(x(t), y(t)) d t, x, y \in L^{2}$. Символ $\mathscr{F}=\mathscr{F}(\mathbb{R}, \mathscr{H})$ используется для обозначения одного из пространств $C_{b}(\mathbb{R}, \mathscr{H}), L^{p}(\mathbb{R}, \mathscr{H}), p \in$ $[1, \infty]$.

Слабым ограниченным решением уравнения (1.1), где $f \in \mathscr{F}(\mathbb{R}, \mathscr{H})$, называется функция $x \in C_{b} \cap \mathscr{F}$ такая, что при всех $s \leqslant t$ из $\mathbb{R}$ верны равенства

$$
x(t)=T(t-s) x(s)+\int_{s}^{t} T(t-\tau) f(\tau) d \tau, \quad s \leqslant t .
$$

Важной является следующая задача: найти необходимые и достаточные условия на оператор $A$, обеспечивающие существование и единственность (слабого) решения $x \in C_{b} \cap \mathscr{F}$ для любой функции $f \in \mathscr{F}$. Эта задача эквивалентна непрерывной обратимости дифференциального оператора

$$
\mathscr{L}=\mathscr{L}_{\mathscr{F}}=\frac{d}{d t}-A: D(\mathscr{L}) \subset \mathscr{F} \rightarrow \mathscr{F}=\mathscr{F}(\mathbb{R}, \mathscr{H})
$$

определяемого следующим образом. Функция $x \in C_{b} \cap \mathscr{F}$ считается принадлежащей области определения $D\left(\mathscr{L}_{\mathscr{F}}\right)$ оператора, если существует функция $f \in \mathscr{F}$ такая, что $x$ - слабое решение дифференциального уравнения (1.1), т.е. верны равенства (1.2). Оператор $\mathscr{L}_{\mathscr{F}}$ называется непрерывно обратимым, если его ядро $\operatorname{Ker} \mathscr{L}_{\mathscr{F}}$ нулевое и образ $\operatorname{Im} \mathscr{L}_{\mathscr{F}}$ совпадает с $\mathscr{F}$. Следовательно, $\mathscr{L}_{\mathscr{F}}^{-1} \in$ End $\mathscr{F}$.

ОПредЕлЕниЕ 1. Полугруппа операторов $T: \mathbb{R}_{+} \rightarrow$ End $\mathscr{H}$ называется гuперболической, или допускающей экспоненииалъную дихотомию, если спектр $\sigma(T(1))$ оператора $T(1)$ обладает свойством

$$
\sigma(T(1)) \cap \mathbb{T}=\varnothing
$$

где $\mathbb{T}=\{\lambda \in \mathbb{C}:|\lambda|=1\}-$ единичная окружность.

Следующий результат содержится в работах [2]-[5].

Теорема 1. Для непрерывной обратимости оператора $\mathscr{L}: D(\mathscr{L}) \subset \mathscr{F} \rightarrow \mathscr{F}$ необходимо и достаточно, чтобы полугруппа $T$ была гиперболической.

Следующая теорема 2 была доказана Прюссом (см. [6], [7]) для полугрупп операторов класса $C_{0}$ (см. также [8] и [9] для вырожденных полугрупп операторов, где в качестве оператора $A$ выступает линейное отношение [9]).

ТЕОРема 2. Для того чтобъ (сильно непрерывная) полугруппа операторов $T: \mathbb{R}_{+} \rightarrow$ End $\mathscr{H}$ была гиперболической, необходимо и достаточно, чтобъ выполнялись следующие два условия:

$$
\begin{gathered}
\sigma(A) \cap(i \mathbb{R})=\varnothing, \\
\sup _{\lambda \in \mathbb{R}}\|R(i \lambda, A)\|<\infty,
\end{gathered}
$$

где $R(\cdot, A): \rho(A) \subset \mathbb{C} \rightarrow \mathscr{H}$ - резольвента оператора $A, \rho(A)=\mathbb{C} \backslash \sigma(A)$ - его резольвентное множество. 
Условие гиперболичности полугруппы операторов $T: \mathbb{R}_{+} \rightarrow$ End $\mathscr{H}$ приводит к разложению

$$
\sigma(T(1))=\sigma_{\text {int }} \cup \sigma_{\text {out }},
$$

где $\sigma_{\text {int }}=\{\lambda \in \sigma(T(1)):|\lambda|<1\}, \sigma_{\text {out }}=\{\lambda \in \sigma(T(1)):|\lambda|>1\}$. Рассмотрим проектор Рисса (см. [1]-[3]),

$$
P_{\text {int }}=\frac{1}{2 \pi i} \int_{\mathbb{T}}(T(1)-\lambda I)^{-1} d \lambda,
$$

построенный по множеству $\sigma_{\text {int }}$ и положим $P_{\text {out }}=I-P_{\text {int }}$, где $I$ - тождественный оператор из алгебры End $\mathscr{H}$. Тогда гильбертово пространство $\mathscr{H}$ представимо в виде прямой суммы

$$
\mathscr{H}=\mathscr{H}_{\text {int }} \oplus \mathscr{H}_{\text {out }}
$$

замкнутого линейного подпространства $\mathscr{H}_{\text {int }}=\operatorname{Im} P_{\text {int }}$, являющегося образом проектора $P_{\text {int }}$, и подпространства $\mathscr{H}_{\text {out }}=\operatorname{Im} P_{\text {out }}$. Из формулы $(1.7)$ следует, что проекторы $P_{\text {int }}, P_{\text {out }}$ перестановочны с операторами $T(t), t \geqslant 0$, и, следовательно, подпространства $\mathscr{H}_{\text {int }}, \mathscr{H}_{\text {out }}$ инвариантны относительно операторов $T(t), t \geqslant 0$. При этом отметим, что эти подпространства ненулевые, если одновременно $\sigma_{\text {int }} \neq \varnothing, \sigma_{\text {out }} \neq \varnothing$ для множеств из разложения (1.6). Рассмотрим (более подробно см. [4] и [10]) полугруппу операторов

$$
T_{\text {int }}: \mathbb{R}_{+} \rightarrow \text { End } \mathscr{H}_{\text {int }}, \quad T_{\text {int }}(t)=T(t) \mid \mathscr{H}_{\text {int }}, \quad t \geqslant 0,
$$

и группу операторов $T_{\text {out }}: \mathbb{R} \rightarrow$ End $\mathscr{H}_{\text {out }}$, определенную равенствами:

$$
T_{\text {out }}(t)= \begin{cases}T(t) \mid \mathscr{H}_{\text {out }}, & t \geqslant 0, \\ \left(T(-t) \mid \mathscr{H}_{\text {out }}\right)^{-1}, & t<0 .\end{cases}
$$

Tеорема 3 (см. [2]-[4]). Eсли полугруппа T гиперболична, то оператор $\mathscr{L}$ непрерывно обратим и обратный оператор $\mathscr{L}^{-1} \in$ End $\mathscr{F}$ определяется формулой

$$
\left(\mathscr{L}^{-1} f\right)(t)=(G * f)(t)=\int_{\mathbb{R}} G(t-s) f(s) d s, \quad t \in \mathbb{R}, \quad f \in \mathscr{F} .
$$

Функция Грина $G=G_{A}: \mathbb{R} \rightarrow$ End $\mathscr{H}$ имеет вид

$$
G(t)= \begin{cases}T(t) P_{\text {int }}, & t \geqslant 0 \\ -T_{\text {out }}(t) P_{\text {out }}, & t<0\end{cases}
$$

Для гиперболической полугруппы $T$ можно вывести (более подробно см. [2], [4]) существование постоянных $M_{ \pm} \geqslant 1,0<\gamma_{ \pm} \leqslant \infty$, таких, что функция Грина $G$ допускает оценки вида

$$
\|G(u)\|= \begin{cases}M_{+} e^{-\gamma_{+} u}, & u \geqslant 0 \\ M_{-} e^{\gamma_{-} u}, & u<0 .\end{cases}
$$


Отметим, что полагается $\gamma_{-}=\infty$, если полугруппа $T$ экспоненциально устойчива, т.е.

$$
\|T(t)\| \leqslant M_{+} e^{-\gamma_{+} t}, \quad t \geqslant 0
$$

что эквивалентно условию $\sigma_{\text {out }}=\varnothing$ (соответственно полагается $\gamma_{+}=\infty$, если $\sigma_{\text {int }}=\varnothing$.)

Пару чисел $\gamma_{+}, \gamma_{-}$из (1.10) будем называть показателями экспоненииальной дихотомии функции $T$, а две упорядоченных пары чисел $\left(M_{-}, \gamma_{-}\right),\left(M_{+}, \gamma_{+}\right)-$ параметрами экспоненииальной дихотомии. Если полугруппа $T$ экспоненциально устойчива, то пару чисел $\left(M_{+}, \gamma_{+}\right)$назовем параметрами экспоненииальной устойчивости полугруппы $T$.

Из представления (1.8) обратного к $\mathscr{L}$ оператора (при условии (1.3) гиперболичности полугруппы $T$ или условиях (1.4), (1.5)) следует, что норма оператора $\mathscr{L}^{-1}: \mathscr{F} \rightarrow \mathscr{F}=\mathscr{F}(\mathbb{R}, \mathscr{H})$ допускает оценки вида

$$
\left\|\mathscr{L}^{-1}\right\| \leqslant\|G\|_{1}=\int_{-\infty}^{\infty}\|G(\tau)\| d \tau \leqslant \frac{M_{-}}{\gamma_{-}}+\frac{M_{+}}{\gamma_{+}} .
$$

Оценки (1.11) дают возможность получать условия разрешимости в пространстве $C_{b}(\mathbb{R}, \mathscr{H})$ нелинейного уравнения вида

$$
\dot{x}(t)=A x(t)+f(t, x(t)), \quad t \in \mathbb{R},
$$

где $f: \mathbb{R} \times \mathscr{H} \rightarrow \mathscr{H}$ - непрерывная (по первому аргументу) функция равномерно относительно второго аргумента из любого шара из $\mathscr{H}$ и удовлетворяющая условию Липшица по второй переменной:

$$
\|f(t, x)-f(t, y)\| \leqslant \ell\|x-y\|, \quad x, y \in \mathscr{H},
$$

где $\ell>0$ - некоторая постоянная. Тогда имеет место

Теорема 4. Уравнение (1.12) имеет слабое решение $x_{*} \in C_{b}=C_{b}(\mathbb{R}, \mathscr{H})$, если выполнено условие $q=\|G\|_{1} \ell<1$. При этом

$$
\left\|x_{*}\right\|_{\infty} \leqslant \frac{\|G\|_{1}}{1-\|G\|_{1} \ell}\left\|f_{0}\right\|_{\infty}
$$

где $f_{0}(t)=f(t, 0), t \in \mathbb{R}$.

ДокАЗАТЕЛьство аналога теоремы 4 имеется в статье [11]; там же имеется определение слабого решения нелинейного уравнения. Оно основано на применении принципа сжимающих отображений [12] к интегральному уравнению

$$
x(t)=(\Phi(x))(t)=\int_{\mathbb{R}} G(t-s) f(s, x(s)) d s, \quad t \in \mathbb{R},
$$

где $\Phi: C_{b} \rightarrow C_{b}-$ сжимающее отображение с константой сжатия $\left\|\mathscr{L}^{-1}\right\| \ell \leqslant$ $\|G\|_{1} \ell<1$.

Таким образом, из теоремы 4 следует важность получения оценки для $\|G\|_{1}$. В настоящей статье для оценок используются следующие величины (см. также 
формулы из теоремы 8):

$$
\begin{aligned}
\gamma(A) & =\sup _{\lambda \in \mathbb{R}}\|R(i \lambda, A)\|, \\
\nu(A) & =\sup _{\|x\| \leqslant 1} \frac{1}{2 \pi} \int_{\mathbb{R}}\|R(i t, A) x\|^{2} d t, \\
\nu\left(A^{*}\right) & =\sup _{\|x\| \leqslant 1} \frac{1}{2 \pi} \int_{\mathbb{R}}\left\|R\left(i t, A^{*}\right) x\right\|^{2} d t, \\
k_{c}(T) & =\sup _{0 \leqslant t \leqslant c}\|T(t)\|, \quad c>0, \\
\beta(A) & =\sup _{\mu \in \Theta(A)} \operatorname{Re} \mu,
\end{aligned}
$$

где $\Theta(A)=\{(A x, x) ;\|x\| \leqslant 1, x \in D(A)\}$ - числовая область оператора $A$ (см. [13]). Таким образом, в оценках (1.13)-(1.15) используются величины, построенные по резольвенте оператора $A$.

Конечность величины $\gamma(A)$ следует из теоремы 2. Она важна тем, что дает равенство $\left\|\mathscr{L}^{-1}\right\|=\gamma(A)$ для нормы обратного оператора к дифференциальному оператору $\mathscr{L}=\frac{d}{d t}-A: D(\mathscr{L}) \subset L^{2}(\mathbb{R}, \mathscr{H}) \rightarrow L^{2}(\mathbb{R}, \mathscr{H})$. Для доказательства этого равенства в лемме 4 статьи [4] использовалось равенство Парсеваля. Величина $\gamma(A)$ использовалась в работах [14], [4], [15] для оценок норм обратных операторов к дифференциальным операторам. В статье [16] она называлась частотной характеристикой оператора $A$.

В монографии [14] для $A \in$ End $\mathscr{H}$, где $\mathscr{H}$ - конечномерное пространство, использовалась величина $\nu(A)$. Она называлась интегралъным критерием качества дихотомии. В статье [10] установлена оценка $\gamma(A) \leqslant 2 \nu(A)$. Отметим еще (без доказательства) оценку $\gamma(A) \leqslant\left(\nu(A) \nu\left(A^{*}\right)\right)^{1 / 2}$.

Использование величины $\beta(A)$, определяемой равенством (1.17) по числовой области $\Theta(A)$ оператора $A$, является важным в связи со следующей теоремой (см. [17; теорема I.4.2 и п. I.4.4]).

Tеорема 5. Для того чтобъ оператор $A: D(A) \subset \mathscr{H} \rightarrow \mathscr{H}$ с плотной в $\mathscr{H}$ областью определения $D(A)$ был инфинитезимальным оператором полугруппы операторов $T: \mathbb{R}_{+} \rightarrow$ End $\mathscr{H}$, удовлетворяющей оченке

$$
\|T(t)\| \leqslant e^{w t}, \quad t \geqslant 0,
$$

где $w \in \mathbb{R}$, необходимо и достаточно выполнение условий

$$
\begin{aligned}
\operatorname{Re}(A x, x) \leqslant w(x, x), & x \in D(A), \\
\operatorname{Re}\left(A^{*} y, y\right) \leqslant w(y, y), & y \in D\left(A^{*}\right) .
\end{aligned}
$$

Таким образом, выполнение оценки (1.18) эквивалентно выполнению условий $\beta(A) \leqslant w, \beta\left(A^{*}\right) \leqslant w$, т.е. числовые области $\Theta(A)$ и $\Theta\left(A^{*}\right)$ операторов $A, A^{*}$ лежат в полуплоскости $\mathbb{C}_{w}=\{\lambda \in \mathbb{C}: \operatorname{Re} \lambda \leqslant w\}$ (выполнены условия (1.19), (1.20)). Однако не для всякой полугруппы $T: \mathbb{R}_{+} \rightarrow$ End $\mathscr{H}$ найдется $w \in \mathbb{R}$ такое, что верна оценка (1.18). В $\S 4$ приведен пример полугруппы операторов, инфинитезимальный оператор которой и его сопряженный 
оператор имеют числовые области, совпадающие со всей комплексной плоскостью.

В условиях следующей теоремы для инфинитезимального оператора $A$ группы операторов $T: \mathbb{R}_{+} \rightarrow$ End $\mathscr{H}$ делается

ПРЕДПОЛОЖЕНИЕ 1. Величинъ

$$
\beta_{\min }(A)=-\beta(-A)=\inf \{\operatorname{Re} \lambda: \lambda \in \Theta(A)\}, \quad \beta_{\max }(A)=\beta(A)
$$

конечны, $и \beta_{\min }(A)<0, \beta_{\max }(A)>0$.

ЗАмЕчАниЕ 1. Условия предположения 1 выполнены для оператора

$$
A: D(A) \subset \mathscr{H} \rightarrow \mathscr{H}, \quad \overline{D(A)}=\mathscr{H},
$$

если $D\left(A^{*}\right)=D(A)$ и оператор $2^{-1}\left(A+A^{*}\right): D(A) \subset \mathscr{H} \rightarrow \mathscr{H}$ допускает ограниченное расширение на $\mathscr{H}$ до (самосопряженного) оператора $B \in$ End $\mathscr{H}$, спектр $\sigma(B)$ которого удовлетворяет условиям $\sigma(B) \cap \mathbb{R}_{-} \neq \varnothing, \sigma(B) \cap \mathbb{R}_{+} \neq \varnothing$, где $\mathbb{R}_{-}=(-\infty, 0]$. В частности, перечисленным условиям удовлетворяет оператор $A=i A_{0}+B$, где $A_{0}: D\left(A_{0}\right) \subset \mathscr{H} \rightarrow \mathscr{H}$ - самосопряженный оператор и $B=B^{*} \in$ End $\mathscr{H}$ имеет спектр, для которого $\sigma(B) \cap \mathbb{R}_{ \pm} \neq \varnothing$.

Теорема 6. Пусть $T: \mathbb{R} \rightarrow$ End $\mathscr{H}$ - гиперболическая группа операторов, для которой выполнено предположение 1. Тогда функиия Грина $G_{A}: \mathbb{R} \rightarrow$ End $\mathscr{H}$ допускает оценки вида

$$
\begin{gathered}
\left\|G_{A}(t)\right\| \leqslant \begin{cases}\left(2\left|\beta_{\min }(A)\right| \nu(A)\right)^{1 / 2} \exp \left\{-\frac{1}{2 \nu(A)} t\right\}, & t \geqslant 0, \\
\left(2 \beta_{\max }(A) \nu(A)\right)^{1 / 2} \exp \left\{\frac{1}{2 \nu(A)} t\right\}, & t<0,\end{cases} \\
\left\|G_{A}\right\|_{1} \leqslant 2 \nu(A)\left(2 \beta_{\max }(A) \nu(A)\right)^{1 / 2}+\left(2\left|\beta_{\min }(A)\right| \nu(A)\right)^{1 / 2} .
\end{gathered}
$$

СлеДСтвиЕ 1. В условиях теоремы 2 нормы проекторов $P_{\text {int }}, P_{\text {out }}$, участвующих в определении функиии Грина (формула (1.9)), допускают оценки

$$
\max \left\{\left\|P_{\text {int }}\right\|,\left\|P_{\text {out }}\right\|\right\} \leqslant\left(2 \min \left\{\left|\beta_{\min }(A)\right|, \beta_{\max }(A)\right\} \nu(A)\right)^{1 / 2} .
$$

При доказательстве теоремы 6 существенно используется теорема 6 из статьи [10], в которой на основе использования уравнения Ляпунова получено необходимое и достаточное условие гиперболичности групп операторов (см. также монографию [3; теорема 4.40]). Доказательство теоремы 6 ведется на основе построения в гильбертовом пространстве $\mathscr{H}$ индефинитной метрики, задаваемой самосопряженным оператором $W \in$ End $\mathscr{H}$, относительно которого решение соответствующего дифференциального уравнения экспоненциально убывает при $t \rightarrow \infty$. В [10] построен пример гиперболической полугруппы компактных операторов, для которых определяемый из уравнения Ляпунова оператор $W=W^{*} \in$ End $\mathscr{H}$ не является обратимым, т.е. для полугрупп операторов не представляется возможность получения таким способом аналогов 
теоремы 6. Для конечномерного пространства $\mathscr{H}$ аналоги теоремы 6 получены в работах [14], [18]-[22].

Предположим, что $T: \mathbb{R}_{+} \rightarrow$ End $\mathscr{H}$ - экспоненциально устойчивая полугруппа операторов. Тогда (см. [3], [10] и [23]) уравнение Ляпунова

$$
A^{*} X+X A=-I
$$

имеет решение $W=W^{*} \in$ End $\mathscr{H}$, которое определяется формулой

$$
W x=-\int_{0}^{\infty} T(t)^{*} T(t) x d t, \quad x \in \mathscr{H} .
$$

Таким образом, $W D(A) \subset D\left(A^{*}\right)$ и $A^{*} W x+W A x=-x, x \in D(A)$. При этом оператор $W$ отрицательно определен, но не обязательно непрерывно обратим.

Теорема 7. Пусть $T: \mathbb{R}_{+} \rightarrow$ End $\mathscr{H}$ - экспоненииально устойчивая полугруппа операторов и оператор $W$ непрерывно обратим. Тогда имеют место оценки

$$
\|T(t)\| \leqslant \sqrt{\|W\|\left\|W^{-1}\right\|} \exp \left\{-\frac{1}{2\|W\|} t\right\}, \quad t \geqslant 0 .
$$

Отметим, что для экспоненциально устойчивой группы операторов $T: \mathbb{R} \rightarrow$ End $\mathscr{H}$ оператор $W$ обратим (см. [10] и теорему 17). Из замечания 2 (см. 2 ) следует, что $\|W\|=\nu(A)$.

В случае конечномерного пространства $\mathscr{H}$ оценка (1.25) приведена в [14; п. 9.3].

В §3 предложен другой способ получения оценки функции Грина, основанный на использовании методов гармонического анализа.

Наряду с определяемыми формулами (1.13)-(1.17) величинами в теореме 8 вводятся в рассмотрение новые величины $\nu_{\varepsilon}(A), \nu_{\varepsilon}\left(A^{*}\right)$. Они определяются по произвольному линейному оператору $A: D(A) \subset \mathscr{H} \rightarrow \mathscr{H}$, имеющему непустое резольвентное множество $\rho(A)$ и для которого конечны величины

$$
s(A)=\sup \{\operatorname{Re} \lambda: \lambda \in \sigma(A)\}, \quad s_{0}(A)=\inf \left\{a>s(A): \sup _{\operatorname{Re} \lambda \geqslant a}\|R(\lambda, A)\|<\infty\right\} .
$$

Величину $s(A)$ называют (см. [7], [23]) спектральной границей оператора $A$, а величину $s_{0}(A)$ - абсииссой равномерной ограниченности резолъвенты оператора $A$.

Для произвольной (сильно непрерывной) полугруппы операторов $T: \mathbb{R}_{+} \rightarrow$ End $\mathscr{H}$ величина

$$
w_{0}(T)=\inf \left\{w \in \mathbb{R}: \sup _{t \geqslant 0} e^{-w t}\|T(t)\|<\infty\right\}
$$

называется типом полугруппь $T$ или постоянной роста полугруппь $T$ (см. [7], [23]).

Отметим, что $s(A) \leqslant s_{0}(A) \leqslant w_{0}(T)$ для инфинитезимального оператора $A$ полугруппы $T$. При этом $s_{0}(A)=w_{0}(T)$ в силу теоремы 2. Для полугрупп операторов в банаховом пространстве эти величины могут не совпадать [7]. 
Teоpema 8. Onepamop $A: D(A) \subset \mathscr{H} \rightarrow \mathscr{H} c \overline{D(A)}=\mathscr{H}$ является инфинитезимальным оператором экспоненииально устойчивой полугруппы операторов $T: \mathbb{R}_{+} \rightarrow$ End $\mathscr{H}$ тогда и только тогда, когда для некоторого $\varepsilon>0$ выполнены условия:

1) $s_{0}(A)<0$;

2) $\nu_{\varepsilon}(A)=\sup _{a>0} \sup _{\|x\| \leqslant 1} a \int_{-\infty}^{\infty}\left\|R\left(a+s_{0}(A)+\varepsilon+i \lambda, A\right) x\right\|^{2} d \lambda<\infty$;

3) $\nu_{\varepsilon}\left(A^{*}\right)=\sup _{a>0} \sup _{\|x\| \leqslant 1} a \int_{-\infty}^{\infty}\left\|R\left(a+s_{0}(A)+\varepsilon+i \lambda, A^{*}\right) x\right\|^{2} d \lambda<\infty$.

При выполнении этих условий полугруппа $T: \mathbb{R}_{+} \rightarrow$ End $\mathscr{H}$ допускает ощенку

$$
\|T(t)\| \leqslant \frac{e}{2 \pi} \sqrt{\nu_{\varepsilon}(A) \nu_{\varepsilon}\left(A^{*}\right)} \exp \left\{\left(s_{0}(A)+\varepsilon\right) t\right\}, \quad t \geqslant 0
$$

При этом условия 1)-3) будут выполнены для любого $\varepsilon>0$.

Теорема 8 существенно используется в $\S 3$ при доказательстве теоремы 18 , где получены оценки функции Грина для секториальных операторов.

Учитывая трудность получения оценок величин $\nu_{\varepsilon}(A)$ и $\nu_{\varepsilon}\left(A^{*}\right)$, в следующих теоремах используются величины $\gamma(A), \nu(A), \nu\left(A^{*}\right), k_{c}(T)$, заданные формулами (1.13)-(1.16).

Приводимые далее в теоремах 9-11 оценки получены для гиперболической полугруппы операторов $T: \mathbb{R}_{+} \rightarrow$ End $\mathscr{H}$ с инфинитезимальным оператором $A$.

Теорема 9. Функция Грина $G_{A}: \mathbb{R} \rightarrow$ End $\mathscr{H}$ допускает оценки вида

$$
\left\|G_{A}(t)\right\| \leqslant \frac{1}{|t|} \frac{\sqrt{\nu(A) \nu\left(A^{*}\right)}}{(1-\alpha \gamma(A))^{2}} \exp \{-\alpha|t|\}, \quad t \neq 0,
$$

для любого числа $\alpha>0$, удовлетворяющего условию $\alpha<1 / \gamma(A)$. В частности (при $\left.\alpha=(2 \gamma(A))^{-1}\right)$, имеют место оценки

$$
\left\|G_{A}(t)\right\| \leqslant \frac{4}{|t|} \sqrt{\nu(A) \nu\left(A^{*}\right)} \exp \left\{-\frac{|t|}{2 \gamma(A)}\right\}, \quad t \neq 0 .
$$

СлЕДСТвиЕ 2. Если Т - экспоненииально устойчивая полугруппа операторов, то $w_{0}(T) \leqslant(\gamma(A))^{-1}$.

Из теоремы 9 вытекают следующие две теоремы.

Теорема 10. Пусть $T: \mathbb{R}_{+} \rightarrow$ End $\mathscr{H}$ - экспоненциально устойчивая полугруппа операторов. Тогда она допускает ощенки вида

$$
\|T(t)\| \leqslant \begin{cases}k_{c}(T), & 0 \leqslant t \leqslant c \\ \frac{1}{t} \frac{\sqrt{\nu(A) \nu\left(A^{*}\right)}}{(1-\alpha \gamma(A))^{2}} \exp \{-\alpha t\}, & t>c\end{cases}
$$

для любого числа $\alpha>0$, удовлетворяющего условию $\alpha<1 / \gamma(A)$, и для любого $c>0$. 
В следующей теореме для оценки величины $k_{c}(T)$ используется величина $\beta(A)$, определенная равенством (1.17). При этом используется теорема 5.

Tеорема 11. Если $\beta(A)<\infty$ и $T: \mathbb{R}_{+} \rightarrow$ End $\mathscr{H}$ - экспоненииально устойчивая полугруппа операторов, то

$$
\|T(t)\| \leqslant \begin{cases}e^{\beta(A) t}, & 0 \leqslant t \leqslant c \\ \frac{4}{t} \sqrt{\nu(A) \nu\left(A^{*}\right)} \exp \left\{-\frac{1}{2 \gamma(A)} t\right\}, & t>c\end{cases}
$$

для любого числа с >0. При этом

$$
\left\|\mathscr{L}^{-1}\right\| \leqslant\left\|G_{A}\right\|_{1} \leqslant \frac{e^{\beta(A) c}-1}{\beta(A)}+\frac{8}{c} \sqrt{\nu(A) \nu\left(A^{*}\right)} \gamma(A) .
$$

Пусть

$$
\mathscr{L}=\frac{d}{d t}-Q: D(\mathscr{L})=W_{2}^{1}(\mathbb{R}, H) \subset L^{2}(\mathbb{R}, H) \rightarrow L^{2}(\mathbb{R}, H),(\mathbb{R}, H),
$$

где $W^{2,1}(\mathbb{R}, H)=\left\{x \in L^{2}(\mathbb{R}, H): x\right.$ - абсолютно непрерывна и $\left.\dot{x} \in L^{2}(\mathbb{R}, H)\right\}$ пространство Соболева и $Q \in C_{b}(\mathbb{R}$, End $H)$, есть непрерывно обратимый оператор. В статье [15] было доказано, что оператор $\mathscr{L}$ непрерывно обратим в $C_{b}(\mathbb{R}$, End $H)$ и верна оценка

$$
\left\|\mathscr{L}^{-1}\right\|_{\infty} \leqslant 4\left(\left\|\mathscr{L}^{-1}\right\|_{2}+\|Q\|_{\infty}\left\|\mathscr{L}^{-1}\right\|_{2}^{2}\right)
$$

где $\left\|\mathscr{L}^{-1}\right\|_{\infty},\left\|\mathscr{L}^{-1}\right\|_{2}-$ норма оператора $\mathscr{L}^{-1}$ соответственно в $C_{b}$ и $L^{2}$. При доказательстве использовались совершенно другие методы (они затем были использованы в теории вейвлетов [24]).

Результаты $\S 5$ связаны с оценками функции Грина, построенной по гиперболическому линейному отношению. Приведем основные используемые здесь понятия из теории линейных отношений; они изложены в статьях [9], [25]-[27] (см. также монографии [28], [29]).

Пусть $\mathscr{X}$ - комплексное банахово пространство. Любое линейное подпространство $\mathscr{A}$ из декартова произведения $\mathscr{X} \times \mathscr{X}$ называется линейнъм отношением на банаховом пространстве $\mathscr{X}$. Далее любой линейный оператор $A$ такой, что $D(A): \mathscr{X} \rightarrow \mathscr{X}$, отождествляется с линейным отношением

$$
\mathscr{A}=\{(x, A x) \in \mathscr{X} \times \mathscr{X}: x \in D(A)\},
$$

являющимся графиком оператора $A$. Таким образом, имеют место включения

$$
\text { End } \mathscr{X} \subset L O(\mathscr{X}) \subset L R(\mathscr{X}) \text {, }
$$

где $L O(\mathscr{X})$ - множество линейных операторов, действующих в $\mathscr{X}$, а $L R(\mathscr{X})-$ множество линейных отношений на $\mathscr{X}$. Множество замкнутых в $\mathscr{X} \times \mathscr{X}$ линейных отношений обозначим через $L R C(\mathscr{X})$.

Подпространство

$$
D(\mathscr{A})=\{x \in \mathscr{X}: \text { существует } y \in \mathscr{X} \text { такой, что }(x, y) \in \mathscr{A}\}
$$


называется областъю определения отношения $\mathscr{A} \in L R(\mathscr{X})$. Через $\mathscr{A} x$, где $x \in$ $D(A)$, обозначим множество $\{y \in \mathscr{X}:(x, y) \in \mathscr{A}\}$. Подпространства Ker $\mathscr{A}=$ $\{x \in D(\mathscr{A}):(x, 0) \in \mathscr{A}\}, \operatorname{Im} \mathscr{A}=\bigcup_{x \in D(\mathscr{A})} \mathscr{A} x$ называется соответственно ядром и образом отношения $\mathscr{A} \in L R(\mathscr{X})$.

Суммой двух отношений $\mathscr{A}, \mathscr{B} \in L R(\mathscr{X})$ называется линейное подпространство из $\mathscr{X} \times \mathscr{X}$ вида

$$
\mathscr{A}+\mathscr{B}=\{(x, y) \in \mathscr{X} \times \mathscr{X}: x \in D(\mathscr{A}) \cap D(\mathscr{B}), y \in \mathscr{A} x+\mathscr{B} x\},
$$

где $\mathscr{A} x+\mathscr{B} x$ - алгебраическая сумма двух подмножеств $\mathscr{A} x, \mathscr{B} x$. Произведением линейных отношений $\mathscr{A}, \mathscr{B} \in L R(\mathscr{X})$ называется линейное подпространство из $\mathscr{X} \times \mathscr{X}$ вида

$$
\begin{aligned}
\mathscr{A} \mathscr{B}=\{ & (x, z) \in \mathscr{X} \times \mathscr{X}: \\
& \text { существует } y \in D(\mathscr{A}) \text { такой, что }(x, y) \in \mathscr{B},(y, z) \in \mathscr{A}\} .
\end{aligned}
$$

Если $\mathscr{A} \in L R(\mathscr{X})$, то обратное отношение $\mathscr{A}^{-1} \in L R(\mathscr{X})$ определяется равенством $\mathscr{A}^{-1}=\{(y, x) \in \mathscr{X} \times \mathscr{X}:(x, y) \in \mathscr{A}\}$. Ясно, что $D\left(\mathscr{A}^{-1}\right)=\operatorname{Im} \mathscr{A}$ и $\operatorname{Im} \mathscr{A}^{-1}=D(\mathscr{A})$.

Отношение $\mathscr{A}$ из $\operatorname{LRC}(\mathscr{X})$ назовем непрерывно обратимым, если $\mathscr{A}^{-1} \in$ End $\mathscr{X}$, т.е. $\operatorname{Ker} \mathscr{A}=\{0\}$ (отношение $\mathscr{A}$ инъективно) и $\operatorname{Im} \mathscr{A}=\mathscr{X}$ (отношение $\mathscr{A}$ сюръективно). Резольвентным множеством отношения $\mathscr{A}$ называется множество $\rho(\mathscr{A})$ всех $\lambda \in \mathbb{C}$, для которых $(\mathscr{A}-\lambda I)^{-1} \in$ End $\mathscr{X}$, т.е. отношение $\mathscr{A}-\lambda I$ непрерывно обратимо. Спектром отношения $\mathscr{A} \in \operatorname{LRC}(\mathscr{X})$ называется множество $\sigma(\mathscr{A})=\mathbb{C} \backslash \rho(\mathscr{A})$.

Следует отметить, что множество $\rho(\mathscr{A})$ открыто, спектр $\sigma(\mathscr{A})$ отношения $A \in L R C(\mathscr{X})$ замкнут. Функция

$$
R(\cdot, \mathscr{A}): \rho(\mathscr{A}) \rightarrow \text { End } \mathscr{X}, \quad R(\lambda, \mathscr{A})=(\mathscr{A}-\lambda I)^{-1}, \quad \lambda \in \rho(\mathscr{A}),
$$

называется резольвентой отношения $\mathscr{A} \in L R C(\mathscr{X})$.

Замкнутое линейное подпространство $\mathscr{X}_{0} \subset \mathscr{X}$ назовем инвариантным для отношения $\mathscr{A} \in \operatorname{LRC}(\mathscr{X})$ с непустым $\rho(\mathscr{A})$, если оно инвариантно относительно всех операторов $R(\lambda, \mathscr{A}), \lambda \in \rho(\mathscr{A})$. Сужением отношения $\mathscr{A}$ на подпространство $\mathscr{X}_{0}$ назовем отношение $\mathscr{A}_{0} \in L R C\left(\mathscr{X}_{0}\right)$, резольвентой которого является сужение $R_{0}: \rho(\mathscr{A}) \rightarrow$ End $\mathscr{X}_{0}, R_{0}(\lambda)=R(\lambda, \mathscr{A}) \mid \mathscr{X}_{0}, \lambda \in \rho(\mathscr{A})$, peзольвенты $R(\cdot, \mathscr{A}): \rho(\mathscr{A}) \rightarrow$ End $\mathscr{X}$ на подпространство $\mathscr{X}_{0}$ и обозначим его через $\mathscr{A}_{0}=\mathscr{A} \mid \mathscr{X}_{0}$.

Если $\mathscr{X}=\mathscr{X}_{0} \oplus \mathscr{X}_{1}-$ прямая сумма инвариантных относительно $\mathscr{A} \in$ $\operatorname{LRC}(\mathscr{X})$ подпространств и $\mathscr{A}_{0}=\mathscr{A}\left|\mathscr{X}_{0}, \mathscr{A}_{1}=\mathscr{A}\right| \mathscr{X}_{1}$, то будем говорить, что отношение $\mathscr{A}$ является прямой суммой отношений $\mathscr{A}_{0}$ и $\mathscr{A}_{1}$, и использовать запись $\mathscr{A}=\mathscr{A}_{0} \oplus \mathscr{A}_{1}$. Оператор $B \in$ End $\mathscr{X}$ назовем перестановочным с отношением $\mathscr{A} \in L R C(\mathscr{X})$, если $(B x, B y) \in \mathscr{A}$ для любых $(x, y) \in \mathscr{A}$.

ОПределЕниЕ 2. Отношение $\mathscr{A} \in \operatorname{LRC}(\mathscr{X})$ назовем гиперболическим (относительно окружности $\mathbb{T}$ ), если выполнено условие

$$
\sigma(\mathscr{A}) \cap \mathbb{T}=\varnothing
$$


Условие (1.31) для отношения $\mathscr{A} \in L R C(\mathscr{X})$ влечет представление

$$
\sigma(\mathscr{A})=\sigma_{\text {int }} \cup \sigma_{\text {out }},
$$

где $\sigma_{\text {int }}=\{\lambda \in \sigma(\mathscr{A}):|\lambda|<1\}, \sigma_{\text {out }}=\{\lambda \in \sigma(\mathscr{A}):|\lambda|>1\}$. Следующий результат следует из [25] (см. также теорему 4.2 из [26] и теорему 5.2.10 из [27]).

Теорема 12. Пусть $\mathscr{A}$ - гиперболическое линейное отношение на пространстве $\mathscr{X}$. Тогда пространство $\mathscr{X}$ допускает представление в виде

$$
\mathscr{X}=\mathscr{X}_{\text {int }} \oplus \mathscr{X}_{\text {out }},
$$

прямой суммы замкнутых инвариантных относительно линейного отношения $\mathscr{A}$ подпространств $\mathscr{X}_{\text {int }}, \mathscr{X}_{\text {out }}, u \mathscr{A}=\mathscr{A}_{0} \oplus \mathscr{A}_{1}$, где $\mathscr{A}_{0}=\mathscr{A} \mid \mathscr{X}_{\text {int }}$, $\mathscr{A}_{1}=\mathscr{A} \mid \mathscr{X}_{\text {out }} \in \operatorname{LRC}\left(\mathscr{X}_{\text {out }}\right)$ обладают следующими свойствами:

1) $\mathscr{A}_{0} \in$ End $\mathscr{X}_{0}, \sigma\left(\mathscr{A}_{0}\right)=\sigma_{\text {int }}$;

2) $\mathscr{A}_{1} 0=\mathscr{A} 0, D(\mathscr{A})=\mathscr{X}_{\text {int }} \oplus D\left(\mathscr{A}_{1}\right), \sigma\left(\mathscr{A}_{1}\right)=\sigma_{\text {out }}$.

Разложение (1.33) пространства $\mathscr{X}$ осуществляет перестановочный с отношением $\mathscr{A}$ проектор Рисса

$$
P_{0}=-\frac{1}{2 \pi i} \int_{\mathbb{T}} R(\lambda, \mathscr{A}) d \lambda \in \text { End } \mathscr{X} .
$$

При этом $\mathscr{X}_{\text {int }}=\operatorname{Im} P_{0}, \mathscr{X}_{\text {out }}=\operatorname{Im} P_{1}$, где $P_{1}=I-P_{0}$ - дополнительный к $P_{0}$ проектор.

Через $\ell^{p}=\ell^{p}(\mathbb{Z}, \mathscr{X})$, где $p \in[1, \infty]$, обозначим банахово пространство двусторонних последовательностей $x: \mathbb{Z} \rightarrow \mathscr{X}$, для которых конечна величина

$$
\|x\|_{p}=\left(\sum_{n \in \mathbb{Z}}\|x(n)\|^{p}\right)^{1 / p}, \quad p \in[1, \infty), \quad\|x\|_{\infty}=\sup _{n \in \mathbb{Z}}\|x(n)\|, \quad p=\infty,
$$

принимаемая за норму в пространстве $\ell^{p}$.

Рассмотрим разностное включение

$$
x(n) \in A x(n-1)+f(n), \quad n \in \mathbb{Z},
$$

где $f \in \ell^{p}(\mathbb{Z}, \mathscr{X})$ и $A \in L R C(\mathscr{X})$. Под решением этого включения понимается двусторонняя последовательность $x \in \ell^{p}(\mathbb{Z}, \mathscr{X})$, удовлетворяющая (1.35) для каждого $n \in \mathbb{Z}$, т.е. $(x(n-1), x(n)-f(n)) \in A, n \in \mathbb{Z}$. Построим линейное отношение $\mathscr{A} \in L R C\left(\ell^{p}\right)$ на банаховом пространстве $\ell^{p}(\mathbb{Z}, \mathscr{X})$. Оно состоит из пар $(x, y) \in \ell^{p} \times \ell^{p}$ таких, что

$$
(x(k-1), y(k)) \in A, \quad k \in \mathbb{Z} .
$$

Отметим, что замкнутость отношения $A$ влечет замкнутость отношения $\mathscr{A}$. Основываясь на теореме 12 и результатах статьи [30] (где рассматривался случай $A \in L O(\mathscr{X}))$, М. С. Бичегкуев в статье [31] получил следующий результат. 
Теорема 13. Следующие условия эквивалентны:

1) разностное включение (1.35) имеет единственное решение $x \in \ell^{p}$ для любой последовательности $f \in \ell^{p}$;

2) отношение $A$ гиперболично;

3) отношение $I-A$ непрерывно обратимо.

Если выполнено одно из этих условий, то оператор $\mathscr{A}^{-1} \in$ End $\ell^{p}$ допускает представление вида

$$
\left(\mathscr{A}^{-1} f\right)(n)=(G * f)(n)=\sum_{m \in \mathbb{Z}} G(n-m) f(m), \quad n \in \mathbb{Z}, \quad f \in \ell^{p},
$$

где функиия $G: \mathbb{Z} \rightarrow$ End $\mathscr{X}$, называемая функиией Грина для разностного включения (1.35), имеет вид

$$
G(k)= \begin{cases}\mathscr{A}_{0}^{k} P_{0}, & k \geqslant 0, \\ -\mathscr{A}_{1}^{k} P_{1}, & k \leqslant-1,\end{cases}
$$

где $\mathscr{A}_{0}, \mathscr{A}_{1}, P_{0}, P_{1}$ определены в теореме 12 .

Для оценки функции Грина $G$ используется величина

$$
\gamma_{\mathbb{T}}(A)=\sup _{\gamma \in \mathbb{T}}\|R(\gamma, A)\|
$$

Из формулы (1.36) следует, что норма оператора $\mathscr{A}^{-1} \in$ End $\ell^{p}$ допускает оценKy

$$
\left\|\mathscr{A}^{-1}\right\| \leqslant \sum_{k \in \mathbb{Z}}\|G(k)\|
$$

в любом из банаховых пространств $\ell^{p}(\mathbb{Z}, \mathscr{X}), p \in[1, \infty]$.

Теорема 14. Пусть отношение $A \in L R(\mathscr{X})$ гиперболично. Тогда имеет место оченка

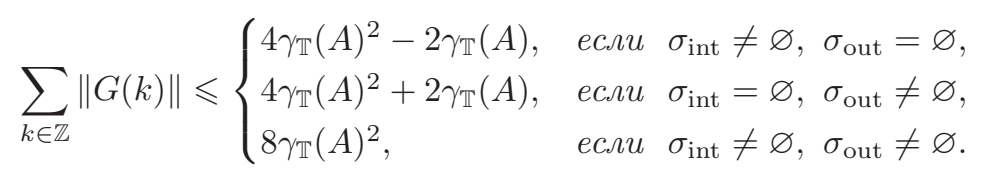

Оценка (1.38) может быть использована в вопросах разрешимости в $\ell^{p}(\mathbb{Z}, \mathscr{X})$ нелинейного разностного включения

$$
x(n) \in A x(n-1)+g(n-1, x(n-1)), \quad n \in \mathbb{Z},
$$

где $A$ - гиперболическое линейное отношение на $\mathscr{X}$ и $g: \mathbb{Z} \times \mathscr{X} \rightarrow \mathscr{X}$ - отображение со свойствами:

1) $\sup _{n \in \mathbb{Z}}\|g(n, m)<\infty\|$ для любого $m \in \mathbb{Z}$,

2) $\sup _{n \in \mathbb{Z}}\|g(n, k)-g(m, \ell)\| \leqslant \ell|k-m|, k \in \mathbb{Z}$, для некоторого $\ell>0$.

Теорема 15. Разностное включение (1.39) разрешимо в пространстве $\ell^{\infty}(\mathbb{Z}, \mathscr{X})$, если выполнено условие

$$
8 \gamma_{\mathbb{T}}(A)^{2} \ell<1
$$

В $\S 6$ приведены примеры гиперболических полугрупп операторов. Приводимые здесь примеры и результаты тесно связаны с результатами статей [32]-[37]. В $§ 7$ обсуждаются некоторые из полученных результатов. 


\section{§ 2. Оценки параметров экспоненциальной дихотомии и функции Грина в случае группы операторов}

Всюду в этом параграфе $T: \mathbb{R}_{+} \rightarrow$ End $\mathscr{H}$ - сильно непрерывная полугруппа операторов. Если она является гиперболической, то используются введенные в 1 обозначения.

Пусть $A$ - инфинитезимальный оператор гиперболической полугруппы операторов $T: \mathbb{R}_{+} \rightarrow$ End $\mathscr{H}$. Рассмотрим в банаховой алгебре End $\mathscr{H}$ уравнение Ляпунова

$$
A^{*} X+X A=F \in \text { End } \mathscr{H} \text {. }
$$

С левой частью этого уравнения свяжем трансформатор (следуя терминологии М. Г. Крейна)

$$
\mathscr{L}: D(\mathscr{L}) \subset \text { End } \mathscr{H} \rightarrow \text { End } \mathscr{H}, \quad \mathscr{L} X=A^{*} X+X A
$$

с областью определения

$$
\begin{aligned}
D(\mathscr{L})=\{ & \left\{X \in \text { End } \mathscr{H}: X x \in D\left(A^{*}\right) \text { для всех } x \in D(A)\right. \\
& \text { и оператор } \left.A^{*} X+X A: D(A) \subset \mathscr{H} \rightarrow \mathscr{H} \text { ограничен на } D(A)\right\} .
\end{aligned}
$$

Если $X \in D(\mathscr{L})$, то в силу плотности $D(A)$ в $\mathscr{H}$ расширение оператора $A^{*} X+$ $X A$ на пространство $\mathscr{H}$ определено корректно и будет обозначаться тем же символом $A^{*} X+X A$.

Введем в рассмотрение замкнутые подпространства из алгебры End $\mathscr{H}$ вида

$$
\begin{aligned}
\operatorname{End}_{*} \mathscr{H} & =\left\{X \in \text { End } \mathscr{H}: P_{\text {int }}^{*} X P_{\text {out }}=P_{\text {out }}^{*} X P_{\text {int }}=0\right\} \\
& =\left\{X \in \text { End } \mathscr{H}: P_{\text {int }}^{*} X P_{\text {int }}+P_{\text {out }}^{*} X P_{\text {out }}=X\right\}, \\
\operatorname{End}_{0} \mathscr{H} & =\left\{X \in \text { End } \mathscr{H}: P_{\text {int }} X P_{\text {out }}^{*}=P_{\text {out }} X P_{\text {int }}^{*}=0\right\} \\
& =\left\{X \in \text { End } \mathscr{H}: P_{\text {int }} X P_{\text {int }}^{*}+P_{\text {out }} X P_{\text {out }}^{*}=X\right\} .
\end{aligned}
$$

Следующие две теоремы были получены в работах [3], [10]. Они существенно используются при доказательстве теорем 6,7 .

Tеорема 16 (см. [3]). Пусть полугруппа $T: \mathbb{R}_{+} \rightarrow$ End $\mathscr{H}$ гиперболична. Тогда уравнение (2.1) разрешимо для любого оператора $F$ из подпространства End $_{*} \mathscr{H}$, причем существует единственное решение $W$, принадлежащее подпространству $\operatorname{End}_{*} \mathscr{H}$. Оно представимо формулой

$$
\begin{aligned}
W x & =-\int_{0}^{\infty} G_{A}(t)^{*} F G_{A}(t) x d t+\int_{\infty}^{0} G_{A}(t)^{*} F G_{A}(t) x d t \\
& =-\int_{0}^{\infty} T(t)^{*} P_{\text {int }}^{*} F T(t) P_{\text {int }} x d t+\int_{\infty}^{0} T(t)^{*} P_{\text {out }}^{*} F T(t) P_{\text {out }} x d t, \quad x \in \mathscr{H} .
\end{aligned}
$$

В частности, если $F=-P_{\text {int }}^{*} P_{\text {int }}-P_{\text {out }}^{*} P_{\text {out }}$, mо оператор $W$ имеет вид

$$
W x=\int_{0}^{\infty} T(t)^{*} P_{\text {int }}^{*} T(t) P_{\text {int }} x d t-\int_{-\infty}^{0} T(t)^{*} P_{\text {out }}^{*} T(t) P_{\text {out }} x d t, \quad x \in \mathscr{H} .
$$


ТЕОРема 17 (см. [10]). Для того чтобы (сильно непрерывная) группа операторов $T: \mathbb{R}_{+} \rightarrow$ End $\mathscr{H}$ была гиперболической, необходимо и достаточно, чтобы существовали равномерно отрищательно определенные операторы $F \ll 0$, $F_{*} \ll 0$ из алгебры End $\mathscr{H}$ такие, что уравнение (2.1) и уравнение

$$
A X+X A^{*}=F_{*}
$$

имели соответственно непрерывно обратимые самосопряженные решения $W \in \operatorname{End}_{*} \mathscr{H}, W_{*} \in \operatorname{End}_{0} \mathscr{H}$. Решение $W$ определяется формулой $(2.2)$, a оператор $W_{*}$ имеет вид

$$
W_{*} x=-\int_{0}^{\infty} G_{A}(t) F_{*} G_{A}(t)^{*} x d t+\int_{\infty}^{0} G_{A}(t) F_{*} G_{A}(t)^{*} x d t, \quad x \in \mathscr{H} .
$$

ЗАмЕчАниЕ 2. В статье [10] было установлено, что для гиперболической полугруппы операторов $T: \mathbb{R}_{+} \rightarrow$ End $\mathscr{H}$ преобразование Фурье $\widehat{G}_{A}: \mathbb{R} \rightarrow$ End $\mathscr{H}$ функции Грина совпадает с сужением резольвенты оператора $A$ на мнимую ось $i \mathbb{R}$, т.е. верны равенства

$$
\widehat{G}_{A}(\lambda) x=\int_{\mathbb{R}} G_{A}(t) x e^{-i \lambda t} d t=R(i \lambda, A) x, \quad \lambda \in \mathbb{R} .
$$

Поэтому из формул (2.2)-(2.5) следует (если использовать тождество Планшереля), что верны равенства

$$
(\widetilde{W} x, x)=\int_{\mathbb{R}}\left\|G_{A}(t) x\right\|^{2} d t=\frac{1}{2 \pi} \int_{\mathbb{R}}\|R(i \lambda, A) x\|^{2} d t, \quad x \in \mathscr{H},
$$

если $F=P_{\text {int }}^{*} P_{\text {int }}-P_{\text {out }}^{*} P_{\text {out }}, \widetilde{W}=W_{+}+W_{-}$, где

$$
W_{+} x=\int_{0}^{\infty} G_{A}(t)^{*} G_{A}(t) x d t, \quad W_{-} x=\int_{\infty}^{0} G_{A}(t)^{*} G_{A}(t) x d t .
$$

Следовательно,

$$
\|\widetilde{W}\|=\sup _{\|x\| \leqslant 1}|(\widetilde{W} x, x)|=\sup _{\|x\| \leqslant 1} \frac{1}{2 \pi} \int_{\mathbb{R}}\|R(i \lambda, A) x\|^{2} d \lambda=\nu(A) .
$$

Пусть $T: \mathbb{R}_{+} \rightarrow$ End $\mathscr{H}$ - (сильно непрерывная) группа операторов. Наряду с группой $T$ рассмотрим группу операторов $\widetilde{T}: \mathbb{R}_{+} \rightarrow$ End $\mathscr{H}, \widetilde{T}(t)=T(-t)$, $t \in \mathbb{R}$. Ее инфинитезимальным оператором является оператор $-A$.

Для доказательства теоремы 6 используется

ЛЕмма 1. Имеют место оценки

$$
\begin{aligned}
& e^{t \beta_{\min }(A)}\|x\| \leqslant\|T(t) x\| \leqslant e^{t \beta_{\max }(A)}\|x\|, \quad t \geqslant 0, \\
& e^{t \beta_{\max }(A)}\|x\| \leqslant\|T(t) x\| \leqslant e^{-t \beta_{\min }(A)}\|x\|, \quad t \leqslant 0, \quad x \in \mathscr{H} .
\end{aligned}
$$

ДокАзАТЕЛьство. Оценки (2.7) (правая часть) следуют из теоремы 5 , где $w=\beta_{\max }(A)$. Поскольку $\beta_{\max }(-A)=-\beta_{\min }(A)$, то

$$
\|x\|=\|T(-t) T(t) x\| \leqslant\|T(-t)\|\|T(t) x\| \leqslant e^{-t \beta_{\min }(A)}\|T(t) x\|, \quad t \geqslant 0 .
$$


Следовательно, имеет место и левая часть оценок (2.7). Оценки (2.8) следуют из оценок (2.7), примененных к полугруппе операторов $\widetilde{T}$. При этом следует учесть равенство $\beta_{\min }(-A)=-\beta_{\max }(A)$. Лемма доказана.

ДокаЗАТЕЛЬСтво теоремЫ 6 . Рассмотрим операторы $W_{+}, W_{-}, \widetilde{W}$ из замечания 2 и будем учитывать, что $\|\widetilde{W}\|=\nu(A)$ (см. (2.6)). Поскольку операторы $W_{+}, W_{-}$равномерно положительны, то

$$
\left(W_{ \pm} x, x\right) \leqslant(\widetilde{W} x, x) \leqslant\|\widetilde{W}\|\|x\|^{2}=\nu(A)\|x\|^{2}, \quad x \in \mathscr{H} .
$$

Из леммы 1 следуют оценки

$$
\begin{aligned}
\left(W_{+} x, x\right) & =\int_{0}^{\infty}\left(T(\tau) P_{\mathrm{int}} x, T(\tau) P_{\mathrm{int}} x\right) d \tau=\int_{0}^{\infty} \|\left(T(\tau) P_{\mathrm{int}} x \|^{2} d \tau\right. \\
& \geqslant \int_{0}^{\infty} e^{2 t \beta_{\min }(A) \tau}\left\|P_{\mathrm{int}} x\right\|^{2} d \tau=\frac{1}{2\left|\beta_{\min }(A)\right|}\left\|P_{\mathrm{int}} x\right\|^{2} \| .
\end{aligned}
$$

Следовательно, имеют место оценки

$$
\left|\left(W_{+} x, x\right)\right| \geqslant \frac{1}{2\left|\beta_{\min }(A)\right|}\left\|P_{\mathrm{int}} x\right\|^{2} \|, \quad x \in \mathscr{H} .
$$

Пусть $x_{0}-$ произвольный вектор из $D(A)$. Рассмотрим функцию $u: \mathbb{R}_{+} \rightarrow \mathscr{H}$, $u(t)=G(t) x_{0}, t \geqslant 0$. При $t \geqslant 0$ получаем равенства

$$
\begin{aligned}
\frac{d}{d t}\left(W_{+} u(t), u(t)\right) & =\frac{d}{d t}\left(P_{\mathrm{int}}^{*} W_{+} P_{\mathrm{int}} u(t), u(t)\right)=\frac{d}{d t}\left(W_{+} P_{\mathrm{int}} u(t), P_{\mathrm{int}} u(t)\right) \\
& =\left(W_{+} P_{\mathrm{int}} A u(t), P_{\mathrm{int}} u(t)\right)+\left(W_{+} P_{\mathrm{int}} u(t), P_{\mathrm{int}} A u(t)\right) \\
& =\left(P_{\mathrm{int}}^{*} W_{+} P_{\mathrm{int}} A u(t), u(t)\right)+\left(P_{\mathrm{int}}^{*} A^{*} W_{+} P_{\mathrm{int}} u(t), u(t)\right) \\
& =\left(\left(A^{*} W_{+}+W_{+} A\right) u(t), u(t)\right)=-\left(P_{\mathrm{int}}^{*} P_{\mathrm{int}} u(t), u(t)\right) \\
& =-\left(P_{\mathrm{int}} u(t), P_{\mathrm{int}} u(t)\right)=-\left\|P_{\mathrm{int}} u(t)\right\|^{2} .
\end{aligned}
$$

Из оценки $(2.9)$ при $x=P_{\text {int }} u(t)$ получаем, что

$$
\begin{aligned}
\left(W_{+} u(t), u(t)\right) & =\left(W_{+} P_{\mathrm{int}} u(t), P_{\mathrm{int}} u(t)\right) \\
& \leqslant\|\widetilde{W}\|\left\|P_{\mathrm{int}} u(t)\right\|^{2}=\nu(A)\left\|P_{\mathrm{int}} u(t)\right\|^{2}, \quad t \geqslant 0 .
\end{aligned}
$$

Таким образом,

$$
\frac{d}{d t}\left(W_{+} u(t), u(t)\right) \leqslant-\frac{1}{\nu(A)}\left(W_{+} u(t), u(t)\right), \quad t \geqslant 0
$$

и, следовательно,

$$
\left(W_{+} u(t), u(t)\right) \leqslant \exp \left\{-\frac{1}{\nu(A)} t\right\}\left(W_{+} u(0), u(0)\right)=\exp \left\{-\frac{1}{\nu(A)} t\right\}\left(W_{+} x_{0}, x_{0}\right) .
$$

Используя оценки (2.9), (2.10), (2.11), получаем

$$
\begin{aligned}
& \frac{1}{2\left|\beta_{\min }(A)\right|}\left\|P_{\text {int }} u(t)\right\|^{2} \leqslant\left(W_{+} u(t), u(t)\right) \leqslant \exp \left\{-\frac{1}{\nu(A)} t\right\}\left(W_{+} x_{0}, x_{0}\right) \\
& \leqslant \exp \left\{-\frac{1}{\nu(A)} t\right\}\left(\widetilde{W} x_{0}, x_{0}\right) \leqslant \nu(A) \exp \left\{-\frac{1}{\nu(A)} t\right\}\left\|x_{0}\right\|^{2}, \quad t \geqslant 0 .
\end{aligned}
$$


Поскольку $u(t)=P_{\mathrm{int}} T(t) x_{0}=G_{A}(t) x_{0}, t \geqslant 0$, и так как $\overline{D(A)}=\mathscr{H}$, то

$$
\left\|G_{A}(t) x\right\|^{2} \leqslant 2\left|\beta_{\min }(A)\right| \nu(A) \exp \left\{-\frac{1}{\nu(A)} t\right\}\|x\|, \quad t \geqslant 0,
$$

для любого вектора $x$ из $\mathscr{H}$. Таким образом,

$$
\left\|G_{A}(t) x\right\| \leqslant\left(2\left|\beta_{\min }(A)\right| \nu(A)\right)^{1 / 2} \exp \left\{-\frac{1}{\nu(A)} t\right\}, \quad t \geqslant 0 .
$$

Оценка $\left\|G_{A}(t)\right\|$ при $t<0$ получается из предыдущей, если рассмотреть группу операторов $\widetilde{T}(t)=T(-t), t \in \mathbb{R}$, при $t \geqslant 0$. Так как генератором является оператор $(-A)$, то $G_{(-A)}(t)=T(-t) P_{\text {оut }}$ при $t>0$. Поскольку $\beta_{\min }(-A)=-\beta_{\max }(A)$, то при $t<0$ получаем, что

$$
\left\|G_{A}(t)\right\|=\left\|G_{-A}(-t)\right\| \leqslant\left(2 \beta_{\max }(A) \nu(A)\right)^{1 / 2} \exp \left\{-\frac{1}{\nu(A)} t\right\}, \quad t<0 .
$$

Из полученных оценок (2.12), (2.13) следуют доказываемые оценки (1.21) и (1.22). Теорема 6 доказана.

ДоКАЗАТЕЛЬСтво тЕОРЕмЫ 7. Пусть $W=W^{*}$ - оператор, определенный формулой (1.24) и удовлетворяющий уравнению Ляпунова (1.23), $x_{0}$ - произвольный вектор из $D(A)$ и $x(t)=T(t) x_{0}, t \geqslant 0$. Имеют место равенства

$$
\begin{aligned}
& \frac{d}{d t}(W x(t), x(t))=(W \dot{x}(t), x(t))+(W x(t), \dot{x}(t)) \\
& \quad=(W A x(t), x(t))+(W x(t), A x(t)) \\
& \quad=\left(\left(W A+A^{*} W\right) x(t), x(t)\right)=-(x(t), x(t))=-\|x(t)\|^{2}, \quad t \geqslant 0 .
\end{aligned}
$$

Из неравенств

$$
(W y, y) \leqslant\|W\|\|y\|^{2}, \quad y \in D(A)
$$

следует, что

$$
\frac{d}{d t}(W x(t), x(t))=-\|x(t)\|^{2} \leqslant-\frac{1}{\|W\|}(W x(t), x(t)), \quad t \geqslant 0 .
$$

Поэтому

$$
(W x(t), x(t)) \leqslant \exp \left\{-\frac{1}{\|W\|} t\right\}\left(W x_{0}, x_{0}\right) \leqslant\|W\| \exp \left\{-\frac{1}{\|W\|} t\right\}\left\|x_{0}\right\|^{2} .
$$

Поскольку

$$
(W x(t), x(t)) \geqslant \frac{1}{\left\|W^{-1}\right\|}\|x(t)\|^{2}, \quad t \geqslant 0
$$

то

$$
\|x(t)\|^{2}=\left\|T(t) x_{0}\right\|^{2} \leqslant\left\|W^{-1}\right\|\|W\| \exp \left\{-\frac{1}{\|W\|} t\right\}\left\|x_{0}\right\|, \quad t \in \mathbb{R}_{+}, \quad x_{0} \in D(A) .
$$


В силу ограниченности полугруппы операторов $T: \mathbb{R}_{+} \rightarrow$ End $\mathscr{H}$ на любом конечном промежутке $[0, r], r>0$, и плотности $D(A)$ в пространстве $\mathscr{H}$ из $(2.14)$ следует, что

$$
\|T(t)\|^{2} \leqslant\left\|W^{-1}\right\|\|W\| \exp \left\{-\frac{1}{\|W\|} t\right\}, \quad t \geqslant 0 .
$$

Из этого неравенства следует оценка (1.25). Теорема доказана.

\section{§ 3. Оценки функции Грина, построенной по гиперболической полугруппе операторов; доказательство теорем 8-11}

ДокАЗАТЕЛЬСтво теоремы 8. Необходимость. Пусть $\widetilde{T}: \mathbb{R}_{+} \rightarrow$ End $\mathscr{H}-$ (сильно непрерывная) ограниченная полугруппа операторов с инфинитезимальным оператором $\widetilde{A}: D(\widetilde{A}) \subset \mathscr{H} \rightarrow \mathscr{H}$. Тогда $s(\widetilde{A}) \leqslant s_{0}(\widetilde{A})=w_{0}(\widetilde{T}) \leqslant 0$.

Для любого числа $a>0$ полугруппа операторов $\widetilde{T}_{a}(t)=e^{-a t} \widetilde{T}(t), t \geqslant 0$, является экспоненциально устойчивой полугруппой операторов с инфинитезимальным оператором $\widetilde{A}-a I$. Тогда из равенства (2.5) следует представление

$$
\begin{aligned}
\widetilde{T}(t) x & =e^{a t} \widetilde{T}_{a}(t) x=-\frac{1}{2 \pi} \int_{-\infty}^{\infty} e^{(a+i \lambda) t} R(-a+i \lambda, \widetilde{A}) x d \lambda \\
& =\frac{e^{a t}}{2 \pi t} \int_{-\infty}^{\infty} e^{i \lambda t} R(-a+i \lambda, \widetilde{A})^{2} x d \lambda, \quad x \in \mathscr{H}, \quad a>0 .
\end{aligned}
$$

Из этого представления для любых $x, y \in \mathscr{H}$ получаем оценки

$$
\begin{aligned}
|(\widetilde{T}(t) x, y)|= & \frac{e^{a t}}{2 \pi t}\left|\int_{-\infty}^{\infty} e^{i \lambda t}\left(R(-a+i \lambda, \widetilde{A}) x, R\left(-a-i \lambda, \widetilde{A}^{*}\right) y\right) d \lambda\right| \\
\leqslant & \frac{e^{a t}}{2 \pi t a} \sup _{\|x\| \leqslant 1}\left(a \int_{-\infty}^{\infty} \| R\left(-a+i \lambda, \widetilde{A} x \|^{2} d \lambda\right)^{1 / 2}\right) \\
& \times\left(\sup _{\|y\| \leqslant 1}\left(a \int_{-\infty}^{\infty}\left\|R\left(-a+i \lambda, \widetilde{A}^{*}\right) y\right\|^{2} d \lambda\right)^{1 / 2} .\right.
\end{aligned}
$$

Для любого числа $\varepsilon>0$ полугруппа операторов

$$
\widetilde{T}_{\varepsilon}: \mathbb{R}_{+} \rightarrow \text { End } \mathscr{H}, \quad \widetilde{T}_{\varepsilon}(t)=e^{\left(-s_{0}(A)-\varepsilon\right) t} T(t), \quad t \geqslant 0,
$$

ограничена. Ее инфинитезимальным оператором является оператор $\widetilde{A}_{\varepsilon}=A-$ $\left(s_{0}(A)+\varepsilon\right) I$. Применяя полученную оценку $(3.1)$ к полугруппе операторов $\widetilde{T}_{\varepsilon}$, получаем, что для любого $a>0$ верны оценки

$$
\begin{aligned}
\left\|\widetilde{T}_{\varepsilon}(t)\right\| & =e^{\left(-s_{0}(A)-\varepsilon\right) t}\|T(t)\| \\
\leqslant & \frac{e^{a t}}{2 \pi t a} \sup _{\|x\| \leqslant 1}\left(a \int_{-\infty}^{\infty}\left\|R\left(a+s_{0}(A)+\varepsilon+i \lambda, A\right) x\right\|^{2} d \lambda\right)^{1 / 2} \\
& \quad \times \sup _{\|x\| \leqslant 1}\left(a \int_{-\infty}^{\infty}\left\|R\left(a+s_{0}(A)+\varepsilon+i \lambda, A\right) x\right\|^{2} d \lambda\right)^{1 / 2}, \quad t>0 .
\end{aligned}
$$


Из этих оценок при $a=t^{-1}$ получаем, что

$$
\left\|T_{\varepsilon}(t)\right\| \leqslant \frac{e}{2 \pi}\left(\nu_{\varepsilon}(A) \nu_{\varepsilon}\left(A^{*}\right)\right)^{1 / 2}, \quad t>0 .
$$

Следовательно,

$$
\begin{aligned}
\|T(t)\| & =e^{\left(s_{0}(A)+\varepsilon\right) t}\left\|T_{\varepsilon}(t)\right\| \\
& \leqslant \frac{e}{2 \pi}\left(\nu_{\varepsilon}(A) \nu_{\varepsilon}\left(A^{*}\right)\right)^{1 / 2} e^{\left(s_{0}(A)+\varepsilon\right) t}, \quad t>0,
\end{aligned}
$$

для любого $\varepsilon>0$. Таким образом, из (3.2) следует оценка (1.26).

Достаточность условий теоремы следует из теоремы Гомилко [38], примененной к полугруппе операторов $\widetilde{T}_{\varepsilon}: \mathbb{R}_{+} \rightarrow$ End $\mathscr{H}$. А именно, выполнение условий 1$)-3$ ) теоремы 8 влечет ограниченность полугруппы $\widetilde{T}_{\varepsilon}$ при любом $\varepsilon>0$. Поэтому полугруппа $T(t)=e^{\left(s_{0}(A)+\varepsilon\right) t} T_{\varepsilon}(t), t \geqslant 0$, будет экспоненциально устойчивой, если положить $\varepsilon=-1 / 2 s_{0}(A)$. Теорема 8 доказана.

Теорема 8 может быть использована для оценки параметров экспоненциальной устойчивости аналитических полугрупп операторов. Их инфинитезимальными операторами являются секториальные операторы (см. [7], [39]).

Оператор $A: D(A) \subset \mathscr{H} \rightarrow \mathscr{H}$ называется секториальным с углом $\Theta \in$ $(\pi / 2, \pi)$, если для некоторого $b \in \mathbb{R}$ сектор

$$
\Omega=\Omega(a, \Theta)=\{\lambda \in \mathbb{C}: \arg (\lambda-a)<\Theta, \lambda \neq a\}
$$

содержится в резольвентном множестве $\rho(A)$ оператора $A$ и для каждого $\delta \in$ $(0, \Theta-\pi / 2)$ существует число $M_{\delta} \geqslant 1$ такое, что

$$
\sup _{\lambda \in \Omega(b, \Theta-\delta)}\|(b-\lambda) R(\lambda, A)\|=M_{\delta}<\infty .
$$

Пусть $A: D(A) \subset \mathscr{H} \rightarrow \mathscr{H}$ - секториальный оператор, спектр которого находится в полуплоскости $\mathbb{C}_{-}=\{\lambda \in \mathbb{C}: \operatorname{Re} \lambda<0\}$. Из свойств секториальных операторов (см. [7], [39]) следует, что оператор $A$ является инфинитезимальным оператором некоторой аналитической полугруппы операторов $T: \mathbb{R}_{+} \rightarrow$ End $\mathscr{H}$, причем имеют место равенства

$$
s(A)=s_{0}(A)=w_{0}(T) .
$$

Сразу отметим, что секториальность оператора $A$ не обеспечивает выполнение условий $(1.19),(1.20)$ теоремы 5. Более того, в $§ 4$ будет построен пример секториального оператора, спектр которого отрицательный. Оператор имеет компактную резольвенту и его числовая область заполняет $\mathbb{C}$.

Поскольку $s(A)<0$, то полугруппа операторов $T$ экспоненциально устойчива. Из условия (3.3) следует, что можно в (3.3) считать $b<0$. Более того, существует постоянная $M>0$, для которой

$$
\|R(\lambda, A)\| \leqslant \frac{M}{\left|\lambda-2^{-1} s(A)\right|}
$$

для всех $\lambda \in \mathbb{C}$, лежащих в полуплоскости

$$
\mathbb{C}_{0}^{+}=\left\{\mu \in \mathbb{C}: \operatorname{Re} \mu>2^{-1} s(A)=2^{-1} s_{0}(A)\right\} .
$$


Tеорема 18. Пусть $A: D(A) \subset \mathscr{H} \rightarrow \mathscr{H}$ - секториальный оператор, для которого $s(A)<0$ и резольвента которого удовлетворяет оценке (3.4) в полуплоскости $\mathbb{C}_{0}^{+}$. Тогда полугруппа $T: \mathbb{R}_{+} \rightarrow$ End $\mathscr{H}$, инфинитезимальньм оператором которой является оператор $A$, допускает оценки

$$
\begin{gathered}
\|T(t)\| \leqslant \frac{e M}{4} \exp \left\{\frac{-s(A)}{2} t\right\}, \quad t>0, \\
\left\|G_{A}\right\|_{1}=\int_{0}^{\infty}\|T(\tau)\| d \tau \leqslant \frac{e M}{2 s(A)} .
\end{gathered}
$$

ДокАЗАТЕЛЬСтво. Для получения оценок используем теорему 8, положив $\varepsilon=2^{-1} s(A)$. Для такого $\varepsilon$ получаем оценку величин $\nu_{\varepsilon}(A)$ и $\nu_{\varepsilon}\left(A^{*}\right)$ :

$$
\begin{aligned}
\nu_{\varepsilon}(A) & =\sup _{a>0} \sup _{\|x\| \leqslant 1} a \int_{-\infty}^{\infty}\left\|R\left(a+\frac{s(A)}{2}+i \lambda, A\right) x\right\|^{2} d \lambda \\
& \leqslant \sup _{a>0} \int_{-\infty}^{\infty} \frac{a M}{a^{2}+\lambda^{2}} d \lambda=\frac{\pi}{2} M .
\end{aligned}
$$

Поскольку

$$
\left\|R\left(a+\frac{s(A)}{2}+i \lambda, A^{*}\right)\right\|=\left\|R\left(a+\frac{s(A)}{2}-i \lambda, A\right)\right\|,
$$

то той же константой $\frac{\pi}{2} M$ оценивается величина $\nu_{\varepsilon}\left(A^{*}\right)$. Теперь оценка $(3.5)$ следует из (1.26), а оценка (3.6) - из (3.5). Теорема доказана.

ДоКАЗАТЕЛЬСТво ТЕОРЕМЫ 9. Используя представление функции $\widehat{G}_{A}$ в виде $(2.5)$ равенства $\frac{d}{d \lambda} R(i \lambda, A)=i R(i \lambda, A)^{2}, \lambda \in \mathbb{R}$, и свойства преобразования Фурье, получаем оценки

$$
\begin{aligned}
& \left|\left(i t G_{A}(t) x, y\right)\right|=\frac{1}{2 \pi}\left|\int_{\mathbb{R}}\left(R(i \lambda, A)^{2} x, y\right) e^{i \lambda t} d \lambda\right| \leqslant \frac{1}{2 \pi} \int_{\mathbb{R}}\left|\left(R(i \lambda, A)^{2} x, y\right)\right| d \lambda \\
& \quad=\frac{1}{2 \pi} \int_{\mathbb{R}}\left|\left(R(i \lambda, A) x, R\left(-i \lambda, A^{*}\right) y\right)\right| d \lambda \leqslant \frac{1}{2 \pi} \int_{\mathbb{R}}\|R(i \lambda, A) x\|\left\|R\left(-i \lambda, A^{*} y\right)\right\| d \lambda \\
& \quad \leqslant\left(\frac{1}{2 \pi} \int_{\mathbb{R}}\|R(i \lambda, A) x\|^{2} d \lambda\right)^{1 / 2}\left(\frac{1}{2 \pi} \int_{\mathbb{R}}\left\|R\left(i \lambda, A^{*}\right) y\right\|^{2} d \lambda\right)^{1 / 2} \\
& \leqslant \sqrt{\nu(A) \nu\left(A^{*}\right)}\|x\|\|y\|, \quad x, y \in \mathscr{H}, \quad t>0 .
\end{aligned}
$$

Учитывая произвольность векторов $x, y \in \mathscr{H}$, положим $y=i t G_{A}(t) x$. Тогда

$$
\left\|i t G_{A}(t) x\right\|^{2} \leqslant \sqrt{\nu(A) \nu\left(A^{*}\right)}\|x\|\left\|i t G_{A}(t) x\right\|, \quad x \in \mathscr{H} .
$$

Следовательно, имеют место доказываемые оценки (1.27), (1.28). Теорема доказана.

Теорема 10 (оценки (1.29)) непосредственно следует из теоремы 9.

Теорема 11 (оценки (1.30)) следует из теорем 5 и 9. 


\section{§ 4. О числовой области инфинитезимального оператора полугруппы операторов}

В связи с теоремой 6 важную роль играет вопрос оценок величин $\beta_{\min }(A)$, $\beta_{\max }(A)$ для инфинитезимального оператора $A$ полугруппы операторов $T$ : $\mathbb{R}_{+} \rightarrow$ End $\mathscr{H}$. Эти величины могут быть бесконечными, и, более того, здесь будет построен пример полугруппы операторов, инфинитезимальный оператор которой имеет компактную резольвенту и линейные комбинации собственных векторов которого плотны во всем пространстве. В этом примере числовая область инфинитезимального оператора будет заполнять всю комплексную плоскость $\mathbb{C}$.

Пример 1. Полугруппа операторов $T: \mathbb{R}_{+} \rightarrow$ End $\mathscr{H}$, где $\mathscr{H}=L^{2}(\mathbb{R})$, вида

$$
(T(t) x)(s)= \begin{cases}2 x(s+t), & s \in[-t, 0], \\ x(s+t), & s \in \mathbb{R} \backslash[-t, 0],\end{cases}
$$

такова, что $\beta_{\max }(A)=\infty$ для ее инфинитезимального оператора $A$. Этот факт следует из равенств $\|T(t)\|=2, t \in \mathbb{R}$, и теоремы 5 .

ПримеР 2. Рассмотрим гильбертово пространство $\mathscr{H}=\bigoplus_{n \geqslant 2} \mathbb{C}^{n}$ последовательностей $x=\left(x_{2}, x_{3}, \ldots\right)$, где $x_{n} \in \mathbb{C}^{n}, n \geqslant 2$, со скалярным произведением $\langle x, y\rangle=\sum_{n=2}^{\infty}\left(x_{n}, y_{n}\right), x_{n}, y_{n} \in \mathbb{C}^{n}$. Определим последовательность $\mathscr{N}_{n} \in$ End $\mathbb{C}^{n}, n \geqslant 2$, нильпотентных операторов равенствами:

$$
\mathscr{N}_{n} x=\left(\sum_{k=2}^{n} x_{k}, 0, \ldots, 0\right) \in \mathbb{C}^{n}, \quad n \geqslant 2 .
$$

Индекс нильпотентности каждого оператора $\mathscr{N}_{n}$ равен двум, т.е. $\mathscr{N}_{n}^{2}=0$. Рассмотрим семейство векторов $x(\alpha)=\left(x_{1}(\alpha), \ldots, x_{n}(\alpha)\right) \in \mathbb{C}^{n}, \alpha \in \mathbb{T}$, где

$$
x_{1}(\alpha)=\frac{1}{\sqrt{2}}, \quad x_{k}(\alpha)=\frac{\alpha}{\sqrt{2}} \frac{1}{\sqrt{n-1}}, \quad 2 \leqslant k \leqslant n, \quad\|x(\alpha)\|=1 .
$$

Числовая область $\Theta\left(\mathscr{N}_{n}\right)$ нильпотентного оператора $\mathscr{N}_{n}$ содержит множество комплексных чисел вида

$$
\left(\mathscr{N}_{n} x(\alpha), x(\alpha)\right)=\left\{\sum_{k=2}^{n} x_{k}(\alpha) \overline{x_{1}(\alpha)} ; \alpha \in \mathbb{T}\right\}=\left\{\frac{\alpha \sqrt{n-1}}{2} ; \alpha \in \mathbb{T}\right\} .
$$

Таким образом,

$$
\Theta\left(\mathscr{N}_{n}\right) \supset\left\{\lambda \in \mathbb{C}:|\lambda|=\frac{1}{2} \sqrt{n-1}\right\}=\mathbb{T}\left(\frac{1}{2} \sqrt{n-1}\right)
$$

- окружность радиуса $\frac{1}{2} \sqrt{n-1}$. В силу теоремы Хаусдорфа [13] множество $\Theta\left(\mathscr{N}_{n}\right)$ выпукло и поэтому содержит круг

$$
\mathbb{D}\left(\frac{1}{2} \sqrt{n-1}\right)=\left\{\lambda \in \mathbb{C}:|\lambda| \leqslant \frac{1}{2} \sqrt{n-1}\right\} .
$$


Далее рассмотрим операторы

$$
A_{n}=-\frac{\sqrt{n-1}}{4} I_{n}+\mathscr{N}_{n}, \quad n \geqslant 2,
$$

где $I_{n}$ - тождественный оператор в $\mathbb{C}^{n}$. По ним построим полугруппу операторов $T: \mathbb{R}_{+} \rightarrow$ End $\mathscr{H}$ формулой

$$
T(t) x=\left(e^{t A_{2}} x_{2}, e^{t A_{2}} x_{3}, \ldots\right), \quad x=\left(x_{n}\right) \in \mathscr{H}, \quad t \geqslant 0
$$

Поскольку

$$
e^{t A_{n}}=\exp \left\{-\frac{\sqrt{n-1}}{4} t\right\}\left(I+\mathscr{N}_{n}\right), \quad t \geqslant 0,
$$

то

$$
\left\|e^{t A_{n}}\right\| \leqslant \exp \left\{-\frac{\sqrt{n-1}}{4} t\right\}\left(1+\frac{\sqrt{n-1}}{2} t\right), \quad t \geqslant 0, \quad n \geqslant 2 .
$$

Так как

$$
\begin{aligned}
\|T(t) x\| & =\left(\sum_{n \geqslant 2}\left\|e^{t A_{n}} x_{n}\right\|^{2}\right)^{1 / 2} \leqslant \sup _{n \geqslant 0}\left\|e^{t A_{n}}\right\|\left(\sum_{n \geqslant 0}\left\|x_{n}\right\|^{2}\right)^{1 / 2} \\
& \leqslant \max _{a \geqslant 0} \exp \left\{\frac{-a t}{4}\right\}\left(1+\frac{a t}{2}\right)\|x\|=\frac{2}{\sqrt{e}}\|x\|, \quad t \geqslant 0, \quad x \in \mathscr{H},
\end{aligned}
$$

то полугруппа операторов $T$ ограничена, причем

$$
\sup _{n \geqslant 0}\|T(t)\| \leqslant \frac{2}{\sqrt{e}} .
$$

Нетрудно видеть, что $\|T(t)\| \leqslant M e^{-t / 4}, t \geqslant 0$, т.е. построенная полугруппа экспоненциально устойчива. Ввиду того, что полугруппа $T$ непрерывна на каждом конечномерном подпространстве $\mathscr{H}_{n}=\left\{x=\left(x_{k}\right) \in \mathscr{H}: x_{k}=0, k \geqslant\right.$ $n+1\}, n \geqslant 2$, и эта система подпространств плотна в $\mathscr{H}$, то из ограниченности полугруппы операторов $T$ следует ее сильная непрерывность на $\mathscr{H}$.

Пусть $A$ - инфинитезимальный оператор построенной полугруппы операторов $T: \mathbb{R}_{+} \rightarrow$ End $\mathscr{H}$. Так как подпространства $\mathscr{H}_{n}, n \geqslant 2$, являются инвариантными для $A$, то числовая область $W_{n}$ сужения $A \mid \mathscr{H}_{n}$ оператора $A$ на $\mathscr{H}_{n}$ содержится в числовой области $\Theta(A)$ оператора $A$. Поскольку

$$
W_{n} \supset \bigcup_{k=2}^{n} \Theta\left(A_{k}\right) \supset \bigcup_{k=2}^{n}\left\{\lambda \in \mathbb{C}:\left|\lambda+\frac{\sqrt{k-1}}{4}\right| \leqslant \frac{\sqrt{k-1}}{2}\right\}
$$

(использовалось равенство $\left.\Theta\left(A_{k}\right)=-\sqrt{k-1} / 4+\Theta\left(\mathscr{N}_{k}\right)\right)$ и так как множество

$$
\bigcup_{k=2}^{\infty}\left\{\lambda \in \mathbb{C}:\left|\lambda+\frac{\sqrt{k-1}}{4}\right| \leqslant \frac{\sqrt{k-1}}{2}\right\}
$$

совпадает с $\mathbb{C}$, то $\Theta(A)=\mathbb{C}$. Из определения оператора $A$ следует, что собственные и присоединенные векторы этого оператора порождают пространство $\mathscr{H}$, причем оператор $A$ имеет компактную резольвенту. 
Рассмотрим теперь последовательность операторов $\widetilde{A}_{n}=A_{n}+\Lambda_{n} \in$ End $\mathbb{C}^{n}$, $n \geqslant 2$, где $\Lambda_{n} x=\left(x_{k} / k\right), x=\left(x_{1}, \ldots, x_{n}\right) \in \mathbb{C}^{n}$. Определим полугруппу операторов $\widetilde{T}: \mathbb{R}_{+} \rightarrow$ End $\mathscr{H}$ равенствами

$$
\widetilde{T}(t) x=\left(e^{t \widetilde{A}_{2}} x_{2}, e^{t \widetilde{A}_{3}} x_{3}, \ldots\right) \quad x=\left(x_{n}\right) \in \mathscr{H}, \quad t \geqslant 0 .
$$

Ее инфинитезимальным оператором является оператор $\widetilde{A}=A+\Lambda: D(A) \subset$ $\mathscr{H} \rightarrow \mathscr{H}$, где $\Lambda \in$ End $\mathscr{H}, \Lambda x=\left(\Lambda_{n} x_{n}\right), x=\left(x_{n}\right) \in \mathscr{H}$. Эта полугруппа операторов сильно непрерывна (так как $\widetilde{A}$ есть ограниченное возмущение оператора $\underset{\sim}{A})$, числовая область $\Theta(\widetilde{A})$ оператора $\widetilde{A}$ совпадает с $\mathbb{C}$, резольвента оператора $\widetilde{A}$ компактна, а линейная оболочка собственных векторов оператора $\widetilde{A}$ плотна в пространстве $\mathscr{H}$ (присоединенных векторов оператор $\widetilde{A}$ не имеет).

\section{§5. Доказательство теорем 14,15}

Пусть $A \in L R C(\mathscr{X})$ - гиперболическое отношение на банаховом пространстве $\mathscr{X}$, т.е. выполнено условие (1.31). Поэтому спектр $\sigma(A)$ отношения $A$ представим в виде (1.32). Рассмотрим величины $r_{\text {int }} \geqslant 0,0 \leqslant r_{\text {out }} \leqslant \infty$, положив $r_{\text {int }}=0$, если $\sigma_{\text {int }}=\varnothing$, и $r_{\text {int }}=\max \left\{|\lambda| ; \lambda \in \sigma_{\text {int }}\right\}=r\left(A_{0}\right)$, если $\sigma_{\text {int }} \neq \varnothing$. Положим $r_{\text {out }}=\infty$, если $\sigma_{\text {out }}=\varnothing$, и $r_{\text {out }}=\max \left\{|\lambda| ; \lambda \in \sigma_{\text {out }}\right\}$, если $\sigma_{\text {out }} \neq \varnothing$.

Резольвента $R(\cdot, A): \rho(A) \rightarrow$ End $\mathscr{X}$ отношения $A$ является псевдорезольвентой (см. [1]), которая в силу условия (1.31) теоремы 14 является голоморфной на открытом множестве $\left\{\lambda \in \mathbb{C}: r_{\text {int }}<\rho<r_{\text {out }}\right\}$. Поэтому, используя функциональное исчисление для линейных отношений [25] или теорему 5.9.3 из [1] (см. также доказательство теоремы 6.1 из [27]) для псевдорезольвент, получаем представление

$$
G(n)=\frac{1}{2 \pi i} \int_{\mathbb{T}(\rho)} \gamma^{n} R(\gamma, A) d \gamma, \quad n \in \mathbb{Z},
$$

где $\mathbb{T}(\rho)=\{\lambda \in \mathbb{C}:|\lambda|=\rho\}-$ окружность радиуса $\rho$, удовлетворяющего условию $r_{\text {int }}<\rho<r_{\text {out }}$.

При $n=0$ получаем формулу (см. также формулу (1.34))

$$
P_{0}=-\frac{1}{2 \pi i} \int_{\mathbb{T}(\rho)} R(\gamma, A) d \gamma
$$

для проектора Рисса $P_{0} \in$ End $\mathscr{X}$, построенного по спектральному множеству $\sigma_{\text {int }}$.

Вначале предположим, что $\sigma_{\mathrm{int}} \neq \varnothing$, и оценим нормы $\|G(n)\|$ операторов $G(n), n \leqslant 0$.

Из представления (1.37) функции Грина $G$ следует, что

$$
\|G(n)\| \leqslant \rho^{n+1} \max _{\gamma \in \mathbb{T}}\|R(\gamma \rho, A)\|, \quad n \leqslant 0,
$$

где $\rho \in\left(r_{\text {int }}, 1\right)$. Представим отношение $A-\gamma \rho I$, где $\gamma \in \mathbb{T}, \rho \in\left(r_{\text {int }}, 1\right)$, в следующем виде:

$$
A-\gamma \rho I=(A-\gamma I)(I-(-\gamma)(1-\rho) R(\gamma, A))
$$


Так как оператор

$$
C=(-\gamma)(1-\rho) R(\gamma, A)
$$

удовлетворяет оценке $\|C\| \leqslant(1-\rho) \gamma_{\mathbb{T}}(A), \gamma \in \mathbb{T}$, то $\|C\|<1$ для $\rho \in(1-$ $\left.1 / \gamma_{\mathbb{T}}(A), 1\right)$. Поскольку спектральный радиус ограниченного оператора не превосходит его нормы, то выполнено неравенство

$$
\begin{aligned}
\gamma_{\mathbb{T}}(A) & =\sup _{\gamma \in \mathbb{T}}\|R(\gamma, A)\| \geqslant \sup _{\gamma \in \mathbb{T}} r(R(\gamma, A)) \\
& =\sup _{\gamma \in \mathbb{T}, \lambda \in \sigma(A)} \frac{1}{|\gamma-\lambda|}=\frac{1}{\operatorname{dist}(\mathbb{T}, \sigma(A))} \geqslant \frac{1}{\operatorname{dist}\left(\mathbb{T}, \sigma_{\text {int }}\right)}=\frac{1}{1-r_{\text {int }}} .
\end{aligned}
$$

При этом использовалось равенство

$$
\sigma(R(\gamma, A))=\left\{\frac{1}{\lambda-\gamma} ; \lambda \in \sigma(A)\right\}
$$

которое следует из теоремы 2.4 статьи [25]. Следовательно, $r_{\mathrm{int}} \leqslant 1-1 / \gamma_{\mathbb{T}}(A)$ и $\gamma_{\mathbb{T}}(A) \geqslant 1$.

Тогда из равенства (5.2) следует, что отношение $A-\gamma \rho I$ будет непрерывно обратимо, причем $(A-\gamma \rho I)^{-1}=(I-C)^{-1} R(\gamma, A)$ и имеет место оценка

$$
\begin{aligned}
\|R(\gamma \rho, A)\| & =\|R(\gamma, A)\|\left\|(I-C)^{-1}\right\| \\
& \leqslant \gamma_{\mathbb{T}}(A) \sum_{k=0}^{\infty}(1-\rho)^{k} \gamma_{\mathbb{T}}(A)^{k}=\frac{\gamma_{\mathbb{T}}(A)}{1-(1-\rho) \gamma_{\mathbb{T}}(A)}
\end{aligned}
$$

для всех $\gamma \in \mathbb{T}$ и $\rho \in\left(1-1 / \gamma_{\mathbb{T}}(A), 1\right)$. Поэтому

$$
\begin{aligned}
\sum_{k \leqslant 0}\|G(k)\| & \leqslant \inf _{\rho \in\left(1-1 / \gamma_{\mathbb{T}}(A), 1\right)} \sum_{k=0}^{\infty} \frac{\rho \gamma_{\mathbb{T}}(A)}{1-(1-\rho) \gamma_{\mathbb{T}}(A)} \rho^{k} \\
& =\inf _{\rho \in\left(1-1 / \gamma_{\mathbb{T}}(A), 1\right)} \frac{\gamma_{\mathbb{T}}(A)}{(1-\rho)-(1-\rho)^{2} \gamma_{\mathbb{T}}(A)} .
\end{aligned}
$$

Точная нижняя грань достигается в точке $\rho_{\min }=\sqrt{1-1 / \gamma_{\mathbb{T}}(A)}$. Чтобы избежать громоздкого выражения для оценки, положим $\rho=1-1 /\left(2 \gamma_{\mathbb{T}}(A)\right)$ в правой части оценки (5.4). Тогда верна оценка

$$
\sum_{k \leqslant 0}\|G(k)\|<4 \gamma_{\mathbb{T}}(A)^{2}-2 \gamma_{\mathbb{T}}(A) .
$$

Пусть теперь $\sigma_{\text {out }} \neq \varnothing$. Из представления (5.3), где $1<|\gamma|<r_{\text {out }}$, следует, что

$$
\begin{aligned}
\gamma_{\mathbb{T}}(A) & =\sup _{\gamma \in \mathbb{T}}\|R(\gamma, A)\| \geqslant \sup _{\gamma \in \mathbb{T}} r(R(\gamma, A)) \\
& =\sup _{\gamma \in \mathbb{T}, \lambda \in \sigma(A)} \frac{1}{|\gamma-\lambda|} \geqslant \frac{1}{\operatorname{dist}\left(\mathbb{T}, \sigma_{\text {out }}\right)}=\frac{1}{r_{\text {out }}-1} .
\end{aligned}
$$

Поэтому $r_{\text {out }} \geqslant 1+1 / \gamma_{\mathbb{T}}(A)$. Отношение $A-\gamma \rho I$ запишем в виде

$$
A-\gamma \rho I=(A-\gamma I)(I-(\rho-1) \gamma R(\gamma, A)), \quad \gamma \in \mathbb{T}, \quad \rho \in\left(1, r_{\text {out }}\right) .
$$


Поскольку

$$
(\rho-1)\|R(\gamma, A)\| \leqslant(\rho-1) \gamma_{\mathbb{T}}(A)<\left(r_{\text {out }}-1\right) \gamma_{\mathbb{T}}(A) \leqslant 1,
$$

то отношение $A-\gamma \rho I$ непрерывно обратимо для всех $\gamma \in \mathbb{T}, \rho \in\left(1,1+1 / \gamma_{\mathbb{T}}(A)\right)$ и

$$
R(\gamma \rho, A)=\sum_{n=-\infty}^{-1}((\rho-1) \gamma)^{n} R(\gamma, A)^{n+1}, \quad \gamma \in \mathbb{T}, \quad \rho \in\left(1,1+\frac{1}{\gamma_{\mathbb{T}}(A)}\right)
$$

Следовательно,

$$
\|R(\gamma \rho, A)\| \leqslant \sum_{n=-\infty}^{-1}(\rho-1)^{n} \gamma_{\mathbb{T}}(A)^{n+1}=\frac{\gamma_{\mathbb{T}}(A)}{1-(\rho-1) \gamma_{\mathbb{T}}(A)}
$$

для всех $\gamma \in \mathbb{T}$ и $\rho \in\left(1,1+1 / \gamma_{\mathbb{T}}(A)\right)$. Поэтому из формулы $(5.1)$ получаем оценку

$$
\begin{aligned}
\sum_{k=-\infty}^{-1}\|G(k)\| & \leqslant \inf _{\rho \in\left(1,1+1 / \gamma_{\mathbb{T}}(A)\right)} \sum_{k=0}^{\infty} \frac{\rho^{n+1} \gamma_{\mathbb{T}}(A)}{1-(\rho-1) \gamma_{\mathbb{T}}(A)} \\
& =\inf _{\rho \in\left(1,1+1 / \gamma_{\mathbb{T}}(A)\right)} \frac{\rho \gamma_{\mathbb{T}}(A)}{(\rho-1)\left(1-(\rho-1) \gamma_{\mathbb{T}}(A)\right)} .
\end{aligned}
$$

Положим $\rho=1+1 /\left(2 \gamma_{\mathbb{T}}(A)\right)$, тогда

$$
\sum_{k \geqslant 1}\|G(k)\|<4 \gamma_{\mathbb{T}}(A)^{2}+2 \gamma_{\mathbb{T}}(A)
$$

Из полученных оценок (5.5) и (5.6) следуют доказываемые оценки теоремы. Теорема 14 доказана.

ДоКАЗАТЕЛЬСтво теОРемы 15. Поскольку отношение $\mathscr{A} \in L R C\left(\ell^{\infty}\right)$ непрерывно обратимо, то каждое решение $x_{0} \in \ell^{\infty}(\mathbb{Z}, \mathscr{X})$ нелинейного разностного включения (1.39) есть решение разностного уравнения

$$
x(n)=\sum_{m=-\infty}^{\infty} G(n-m) f(m, x(m))=(\Phi(x))(n), \quad n \in \mathbb{Z} .
$$

Верно и обратное. Определяемое правой частью этого уравнения отображение $\Phi: \ell^{\infty} \rightarrow \ell^{\infty}$ удовлетворяет условию Липшица с постоянной

$$
\sum_{k=-\infty}^{\infty}\|G(k)\| \ell<1
$$

Следовательно, уравнение (5.7) разрешимо в силу принципа сжимающих отображений, а его решение является решением разностного включения (1.39). Теорема доказана. 


\section{§ 6. Об условиях гиперболичности некоторых классов полугрупп операторов. Примеры}

Рассмотрим оператор вида

$$
\mathscr{L}=A-B: D(\mathscr{L})=D(A) \subset \mathscr{H} \rightarrow \mathscr{H},
$$

где $A: D(A) \subset \mathscr{H} \rightarrow \mathscr{H}$ - антисамосопряженный (т.е. $i A-$ самосопряженный) оператор с областью определения $D(A)$ и $B$ - обратимый самосопряженный оператор из алгебры End $\mathscr{H}$. В силу теоремы Стоуна (см. [1; теорема 22.4.3]) оператор $A$ является инфинитезимальным оператором некоторой сильно непрерывной группы изометрических операторов $U: \mathbb{R} \rightarrow$ End $\mathscr{H}$. Следовательно, оператор $\mathscr{L}$ будет инфинитезимальным оператором некоторой группы операторов $T: \mathscr{R} \rightarrow$ End $\mathscr{H}$ класса $C_{0}$ (см. [1; теорема 13.2.1]).

Если $A$ и $B$ - перестановочные операторы, то оператор $A-B$ - нормальный оператор, и поэтому его спектр $\sigma(A-B)$ содержится в множестве

$$
\{\lambda-\mu: \lambda \in \sigma(A) \subset i \mathbb{R}, \mu \in \sigma(B) \subset \mathbb{R}\} .
$$

Следовательно, $\sigma(\mathscr{L}) \cap(i \mathbb{R})=\varnothing$, причем $\operatorname{dist}(\sigma(\mathscr{L}), i \mathbb{R}) \geqslant\left\|B^{-1}\right\|=r\left(B^{-1}\right)=$ $\sup \left\{\alpha^{-1}: \alpha \in \sigma(B)\right\}$. Поэтому $\gamma(\mathscr{L})=\sup _{\lambda \in \mathbb{R}}\|R(i \lambda, \mathscr{L})\| \leqslant\left\|B^{-1}\right\|$. Таким образом, в силу теоремы 2 (выполнены условия (1.4), (1.5)) полугруппа операторов $T$ является гиперболической. Если операторы $A$ и $B$ не перестановочны, то такой результат, вообще говоря, не верен.

Введем в рассмотрение величины и понятия, в терминах которых будет сформулирован результат об условиях гиперболичности рассматриваемой полугруппы операторов $T$.

Рассмотрим два множества

$$
\sigma_{+}=\sigma_{+}(B)=\{\lambda \in \sigma(B): \lambda>0\}, \quad \sigma_{-}=\sigma_{-}(B)=\{\lambda \in \sigma(B): \lambda<0\}
$$

и две величины

$$
æ(B)=æ_{+}(B)+æ_{-}(B), \quad æ(B)=\left\{æ_{-}(B), æ_{+}(B)\right\},
$$

где

$$
æ_{+}(B)=\min \left\{\lambda ; \lambda \in \sigma_{+}\right\}, \quad æ_{-}(B)=\left|\max \left\{\lambda ; \lambda \in \sigma_{-}\right\}\right|,
$$

т.е. $\mathfrak{x}_{ \pm}(B)=\operatorname{dist}\left(\sigma_{ \pm}, i \mathbb{R}\right), \sigma(B)=\sigma_{+} \cup \sigma_{-}$. Далее считается, что оба множества $\sigma_{+}, \sigma_{-}$непусты.

Через $P_{+}=P\left(\sigma_{+}, B\right), P_{-}=P\left(\sigma_{-}, B\right)$ обозначим проекторы Рисса, построенные по множествам $\sigma_{+}$и $\sigma_{-}$соответственно. Ввиду самосопряженности оператора $B$ эти проекторы являются ортогональными.

Наряду с оператором $A$ рассмотрим трансформатор (оператор в пространстве операторов) $\operatorname{ad}_{A}: D\left(\operatorname{ad}_{A}\right) \subset$ End $\mathscr{H} \rightarrow$ End $\mathscr{H}$ с областью определения $D\left(\operatorname{ad}_{A}\right)$, состоящим из таких операторов $X \in$ End $\mathscr{H}$, которые переводят область определения $D(A)$ в область определения $D(A)$; при этом оператор $A X-$ $X A$ допускает расширение с областью определения $D(A)$ до некоторого оператора $Y$ из End $\mathscr{H}$, обозначаемого тем же символом $A X-X A$ или $[A, X]$. При этом полагаем $\operatorname{ad}_{A} X=Y$. 
Теорема 19 (см. [32]). Пусть обратимый оператор В принадлежит области определения $D\left(\operatorname{ad}_{A}\right)$ трансформатора $\operatorname{ad}_{A}$ и выполнено одно из следующих условий:

1) $\|A B-B A\|<æ_{0}(B) \rightsquigarrow(B)$,

2) $\|\left[B, A B-B A \|<æ_{0}(B) æ(B)^{2}\right.$,

3) $\left\|a d_{B}^{n}(A B-B A)\right\|<æ_{0}(B) æ(B)^{n+1}, n \geqslant 2$,

где $а d_{B}^{n}-n$-я степенъ ограниченного трансформатора $\operatorname{ad}_{B}:$ End $\mathscr{H} \rightarrow$ End $\mathscr{H}$, определенного формулой $\operatorname{ad}_{B} X=B X-X B, X \in$ End $\mathscr{H}$. Тогда полугруппа $T$ (инфинитезимальным оператором которой является оператор $\mathscr{L}=\mathscr{A}-\mathscr{B}$ ) гиперболична и имеют место соответствующие (каждому из условий теоремъ) оценки:

$\left.1^{\prime}\right) \gamma(\mathscr{L}) \leqslant \frac{æ(B)}{æ_{0}(B) æ(B)-\|A B-B A\|}$,

$\left.2^{\prime}\right) \gamma(\mathscr{L}) \leqslant \frac{\Re(B)^{2}}{æ_{0}(B) \rightsquigarrow(B)^{2}-\|[B, A B-B A]\|}$,

$\left.3^{\prime}\right) \gamma(\mathscr{L}) \leqslant \frac{\rightsquigarrow(B)^{n+1}}{æ_{0}(B) æ(B)^{n+1}-\left\|a d_{B}^{n}(A B-B A)\right\|}, n \geqslant 2$, где $\gamma(\mathscr{L})=\sup _{\lambda \in \mathbb{R}}\|R(i \lambda, \mathscr{L})\|$.

Важно отметить, что ввиду антисамосопряженности оператора $A$ и самосопряженности оператора $B$ получаем, что $\operatorname{Re}(A x+B x, x)=(B x, x), x \in \mathscr{H}$. Поэтому

$$
\begin{gathered}
\beta_{\min }(A+B)=\min \{\lambda ; \lambda \in \sigma(B)\}, \\
\beta_{\max }(A+B)=\max \{\lambda ; \lambda \in \sigma(B)\}=r(B)=\|B\| .
\end{gathered}
$$

Рассмотрим конкретный пример дифференциального оператора, удовлетворяющего условиям теоремы 19.

Пример 3. Пусть $A=\frac{d}{d t}: D(A)=W_{2}^{1}(\mathbb{R}, H) \subset \mathscr{H} \rightarrow \mathscr{H}-$ оператор дифференцирования, действующий в гильбертовом пространстве $\mathscr{H}=L_{2}(\mathbb{R}, H)$ суммируемых с квадратом нормы функций, определенных на $\mathbb{R}$ со значениями в комплексном гильбертовом пространстве $H$. Напомним, что $\langle x, y\rangle=$ $\int_{\mathbb{R}}(x(t), y(t)) d t, x, y \in \mathscr{H}$ - скалярное произведение в $\mathscr{H}$, если $(\cdot, \cdot)$ - скалярное произведение в $H$. Его областью определения служит пространство Соболева $W_{2}^{1}(\mathbb{R}, H)$, содержащееся в $L_{2}(\mathbb{R}, H)$. Оператор $A$ является инфинитезимальным оператором группы (изометрических) операторов сдвигов функций из $L_{2}(\mathbb{R}, H)$ и поэтому является антисамосопряженным.

Если $B \in$ End $\mathscr{H}$ - оператор умножения на ограниченную непрерывную функцию $Q: \mathbb{R} \rightarrow$ End $\mathscr{H}$, т.е. $(B x)(t)=Q(t) x(t), x \in \mathscr{H}, t \in \mathbb{R}$, то $B$ принадлежит $D\left(\operatorname{ad}_{A}\right)$, если функция $Q$ непрерывно дифференцируема и ее производная $\dot{Q}: \mathbb{R} \rightarrow$ End $\mathscr{H}$ ограничена на $\mathbb{R}$. В этом случае $\operatorname{ad}_{A} B-$ оператор умножения на производную $\dot{Q}$ функции $Q$, т.е.

$$
\left(\left(\operatorname{ad}_{A} B\right) x\right)(t)=\dot{Q}(t) x(t), \quad t \in \mathbb{R} .
$$

Непосредственно из теоремы 19 следует 
Теорема 20. Пусть $Q: \mathbb{R} \rightarrow$ End $H$ - непрерывно дифферениируемая ограниченная функиия с ограниченной производной $\dot{Q}$, значения которой являются самосопряженными обратимыми операторами, и выполнено одно из следующих условий:

1) $\sup _{t \in \mathbb{R}}\|\dot{Q}(t)\|<æ_{0}(Q) æ(Q)$,

$$
\sup \|Q(t) \dot{Q}(t)-\dot{Q}(t) Q(t)\|
$$
где

$$
\begin{aligned}
& x_{+}(Q)=\inf _{t \in \mathbb{R}} \min \left\{\lambda: \lambda \in \sigma(Q(t)) \cap\left(\mathbb{R}_{+}\right\},\right. \\
& x_{-}(Q)=\inf _{t \in \mathbb{R}} \max \left\{|\lambda|: \lambda \in \sigma(Q(t)) \cap\left(\mathbb{R}_{-}\right\}, \quad \text { əде } \quad \mathbb{R}_{-}=(-\infty, 0] .\right.
\end{aligned}
$$

Тогда дифференииальный оператор $\mathscr{L}=\frac{d}{d t}-Q: W_{2}^{1}(\mathbb{R}, H) \subset \mathscr{H} \rightarrow \mathscr{H}$ обратим и обратный оператор допускает соответствуюшую условиям 1), 2) оценку:

$$
\begin{aligned}
& \left.1^{\prime}\right) \quad\left\|\mathscr{L}^{-1}\right\| \leqslant \frac{\Re(Q)}{æ_{0}(Q) \rightsquigarrow(Q)-\sup _{t \in \mathbb{R}}\|\dot{Q}(t)\|}, \\
& \left.2^{\prime}\right) \quad\left\|\mathscr{L}^{-1}\right\| \leqslant \frac{\Re(Q)^{2}}{æ_{0}(Q) \rightsquigarrow(Q)^{2}-\sup _{t \in \mathbb{R}}\|Q(t) \dot{Q}(t)-\dot{Q}(t) Q(t)\|} .
\end{aligned}
$$

Отметим, что из статьи [2] следует, что рассматриваемый дифференциальный оператор $\mathscr{L}=\frac{d}{d t}-Q$ является инфинитезимальным оператором сильно непрерывной группы операторов $T_{u}: \mathbb{R} \rightarrow$ End $\mathscr{H}$, определенной равенствами

$$
\left(T_{u}(t) x\right)(s)=\mathscr{U}(s, s-t) x(s-t), s, t \in \mathbb{R}, \quad x \in \mathscr{H},
$$

где $\mathscr{U}(\tau, s)=U(\tau) U(s)^{-1}, s, \tau \in \mathbb{R}, U: \mathbb{R} \rightarrow$ End $H$ - операторная функция Коши, т.е. $\dot{U}(t)=Q(t) U(t), t \in \mathbb{R}, U(0)=I$ (см. [40; гл. III]). В условиях теоремы 14 полугруппа операторов $T_{u}$ является гиперболической.

Непосредственно из теоремы 20 и сделанного замечания после формулировки теоремы 11 следует

ТеОрема 21. Пусть функиия $Q$ ограничена, непреръвно дифферениируема, $\dot{Q} \in C_{b}(\mathbb{R}$, End $H)$ и выполнено условие 2) теоремы 20. Тогда оператор

$$
\mathscr{L}=\frac{d}{d t}-Q: C_{b}^{1}(\mathbb{R}, H) \subset C_{b} \rightarrow C_{b}=C_{b}(\mathbb{R}, H),
$$

где $C_{b}^{1}(\mathbb{R}, H)=\left\{x \in C_{b}(\mathbb{R}, H): \dot{x} \in C_{b}(\mathbb{R}, H)\right\}$, непрерьвно обратим и его норма $\left\|\mathscr{L}^{-1}\right\|_{\infty}$ допускает оценку

$$
\left\|\mathscr{L}^{-1}\right\|_{\infty} \leqslant 4\left(\varphi(Q)+\|Q\|_{\infty} \varphi(Q)^{2}\right)
$$

где $\varphi(Q)=æ(Q)^{2}\left(æ_{0}(Q) æ(Q)^{2}-\|[Q, \dot{Q}]\|_{\infty}\right),\|[Q, \dot{Q}]\|_{\infty}-$ норма функции $Q \dot{Q}-$ $\dot{Q} Q=[Q, \dot{Q}]{ }_{\text {в }} C_{b}(\mathbb{R}$, End $H)$.

Для оценки величин, определяемых формулами (1.13)-(1.17) и используемых в теоремах $6,7,9-11,18,21$, можно применить метод подобных операторов, изложенный в работах [34]-[37]. Отметим, что метод подобных операторов 
может быть применен для более широкого класса операторов, чем рассматриваемые здесь.

Пусть $A: D(A) \subset \mathscr{H} \rightarrow \mathscr{H}$ - самосопряженный полуограниченный сверху оператор с компактной резольвентой и его спектр $\sigma(A)=\left\{\lambda_{1}, \lambda_{2}, \ldots\right\}$ обладает свойствами

$$
\begin{gathered}
d_{n}=\operatorname{dist}\left(\left\{\lambda_{n}\right\}, \sigma(A) \backslash\left\{\lambda_{n}\right\}\right) \rightarrow \infty, \quad n \rightarrow \infty, \\
\cdots \leqslant \lambda_{n}<\lambda_{n-1}<\cdots<\lambda_{1} .
\end{gathered}
$$

Полуограниченность сверху оператора $A$ означает конечность величины $\beta(A)=$ $\max \left\{\lambda_{n}, n \geqslant 1\right\}=\lambda_{1}$. Пусть $P_{n}, n \geqslant 1,-$ проекторы Рисса (они являются ортопроекторами), построенные по одноточечным множествам $\left\{\lambda_{n}\right\}, n \geqslant 1$. Тогда $A P_{n}=\lambda_{n} P_{n}, n \geqslant 1$, и $x=\sum_{n \geqslant 1} P_{n} x, x \in \mathscr{H}$. Оператор $A$ является секториальным, и, более того, он является инфинитезимальным оператором полугруппы операторов

$$
T_{A}(t) x=\sum_{n \geqslant 1} e^{\lambda_{n} t} P_{n} x, \quad t \geqslant 0, \quad x \in \mathscr{H},
$$

для которой $\left\|T_{A}(t)\right\|=e^{\lambda_{1} t}, t \geqslant 0$. Оператор $i A$ является антисамосопряженным и поэтому является инфинитезимальным оператором группы изометрий

$$
T_{i A}: \mathbb{R} \rightarrow \text { End } \mathscr{H}, \quad T_{i A}(t) x=\sum_{n \in \mathbb{Z}} e^{i \lambda_{n} t} P_{n} x, \quad x \in \mathscr{H}, \quad t \in \mathbb{R} .
$$

По любому натуральному числу $m$ в алгебре $\mathscr{H}$ определим два трансформатора

$$
J_{m}: \text { End } \mathscr{H} \rightarrow \text { End } \mathscr{H}, \quad \Gamma_{m}: \text { End } \mathscr{H} \rightarrow \text { End } \mathscr{H} .
$$

Трансформатор $J_{m}$ (оператор блочной диагонализации) задается формулой

$$
J_{m} X=P_{(m)} X P_{(m)}+\sum_{n \geqslant m+1} P_{n} X P_{n}, \quad X \in \text { End } \mathscr{H}
$$

где сходимость ряда понимается в сильной операторной топологии и проектор $P_{(m)}$ имеет вид: $P_{(m)}=\sum_{k=1}^{m} P_{k}$. Нетрудно показать, что $J_{m}$ - проектор и $\left\|J_{m}\right\|=1$ (см. [36], [37]), при этом ряд для определения $J X$ безусловно сильно сходится. Если образ $\operatorname{Im} P_{n}$ каждого проектора $P_{n}, n \geqslant 1$, одномерен, то $P_{n} x=\left(x, e_{n}\right) e_{n}, n \geqslant 1$, где $e_{n}$ - собственный вектор оператора $A$, отвечающий собственному значению $\lambda_{n}$. В этом случае матрица оператора $Y=J_{m} X$ относительно ортонормированного базиса $\left\{e_{n}, n \geqslant 1\right\}$ будет иметь вид $\left(y_{i j=1}\right)_{i, j}^{\infty}$, где $y_{i j}=\delta_{i j} x_{i j}, \delta_{i j}$ - символ Кронекера, для $\max \{i, j\} \geqslant m+1$ и $y_{i j}=x_{i j}$, если $\max \{i, j\} \leqslant m$. Здесь $x_{i j}=\left(X e_{j}, e_{i}\right), i, j \geqslant 1,-$ матрица оператора $X \in$ End $\mathscr{H}$.

Трансформатор $\Gamma_{m}$ определим, используя трансформатор $\operatorname{ad}_{A}: D\left(\operatorname{ad}_{A}\right) \subset$ End $\mathscr{H} \rightarrow$ End $\mathscr{H}$

$$
\operatorname{ad}_{A} X=A X-X A, \quad X \in D\left(\operatorname{ad}_{A}\right),
$$

с областью определения $D\left(\operatorname{ad}_{A}\right)$, состоящей из операторов $X \in$ End $\mathscr{H}$, обладающих свойствами: 
1) $X D(A) \subset D(A)$,

2) оператор $A X-X A: D(A) \rightarrow \mathscr{H}$ допускает ограниченное расширение $Y$ на пространстве $\mathscr{H}$ (и полагается $\operatorname{ad}_{A} X=Y$ ).

Трансформатор $\Gamma_{m}$ определяется на каждом операторе $X \in$ End $\mathscr{H}$ как (единственное) решение $Y_{m}$ уравнения

$$
A Y-Y A=X-J_{m} X
$$

удовлетворяющее условию $J_{m} Y_{m}=0$. Таким образом, $\left(\Gamma_{m} X\right) D(A) \subset D(A)$ для любого оператора $X \in$ End $\mathscr{H}$. Трансформатор $\Gamma_{m}$ является ограниченным оператором, и имеет место оценка (см. [34], [36])

$$
\left\|\Gamma_{m}\right\| \leqslant \frac{\pi}{2} \max \left\{\alpha_{m}^{-1}, \beta_{m}^{-1}\right\}
$$

где $\alpha_{m}=\operatorname{dist}\left(\sigma_{m}, \sigma(A) \backslash \sigma_{m}\right), \beta_{m}=\min \left\{\lambda_{i}-\lambda_{j}, i \neq j, i, j \geqslant m+1\right\}$. Следовательно, $\lim _{m \rightarrow \infty}\left\|\Gamma_{m}\right\|=0$. Отметим еще, что оператор $\Gamma_{m} X$, где $X \in$ End $\mathscr{H}$, вполне определяется операторами вида (операторными блоками)

$$
P_{i}\left(\Gamma_{m} X\right) P_{j}= \begin{cases}\frac{1}{\lambda_{i}-\lambda_{j}} P_{i} X P_{j}, & \max \{i, j\} \geqslant m+1, i \neq j, \\ 0, & \max \{i, j\} \leqslant m, \\ 0, & i=j .\end{cases}
$$

Следовательно, если $\lambda_{k}, k \geqslant 1,-$ простые собственные значения оператора $A$, то матрица $\left(y_{i j}\right)_{i, j=1}^{\infty}$ оператора $Y=\Gamma X$ имеет вид

$$
y_{i j}= \begin{cases}0, & i, j \leqslant m, \\ \frac{x_{i j}}{\lambda_{i}-\lambda_{j}}, & \max \{i, j\} \geqslant m+1, i \neq j, \\ 0, & i=j,\end{cases}
$$

где $i, j \geqslant 1, x_{i j}=\left(X e_{j}, e_{i}\right), i, j \geqslant 1,-$ матрица оператора $X \in$ End $\mathscr{H}$.

ОПредЕЛЕНиЕ 3. Два линейных оператора $A_{i}: D\left(A_{i}\right) \subset \mathscr{H} \rightarrow \mathscr{H}, i=1,2$, называются подобными, если существует непрерывно обратимый оператор $U \in$ End $\mathscr{H}$ такой, что $U D\left(A_{2}\right)=D\left(A_{1}\right)$ и $A_{1} U x=U A_{2} x, x \in D\left(A_{2}\right)$. Оператор $U$ называется оператором преобразования оператора $A_{1}$ в оператор $A_{2}$.

Подобные операторы обладают рядом совпадающих спектральных свойств. Имеет место следующая лемма, непосредственно вытекающая из определения 3.

Лемма 2. Пусть $A_{i}: D\left(A_{i}\right) \subset \mathscr{H} \rightarrow \mathscr{H}, i=1,2,-$ два подобных оператора u $U \in$ End $\mathscr{H}$ - оператор преобразования оператора $A_{1}$ в $A_{2}$. Тогда:

1) $\sigma\left(A_{1}\right)=\sigma\left(A_{2}\right), \sigma_{d}\left(A_{1}\right)=\sigma_{d}\left(A_{2}\right), \sigma_{c}\left(A_{1}\right)=\sigma_{c}\left(A_{2}\right), \sigma_{r}\left(A_{1}\right)=\sigma_{r}\left(A_{2}\right)$, где $\sigma\left(A_{i}\right), \sigma_{d}\left(A_{i}\right), \sigma_{c}\left(A_{i}\right), \sigma_{r}\left(A_{i}\right), i=1,2$, - соответственно спектр, дискретный, непрерывный и остаточный спектры операторов $A_{i}, i=1,2$;

2) если оператор $A_{2}$ допускает разложение $A_{2}=A_{21} \oplus A_{22}$, где $A_{2 k}=$ $A_{2} \mid \mathscr{H}_{k}, k=1,2,-$ сужение $A_{2}$ на $\mathscr{H}_{k}$ относительно прямой суммы $\mathscr{H}=$ 
$\mathscr{H}_{1} \oplus \mathscr{H}_{2}$ инвариантнъх относительно $A_{2}$ подпространств $\mathscr{H}_{1}, \mathscr{H}_{2}$, то подпространства $\widetilde{\mathscr{H}}_{k}=U\left(\mathscr{H}_{k}\right), k=1,2$, инвариантны относительно оператора $A_{1}$ и $A_{1}=A_{11} \oplus A_{12}$, где $A_{1 k}=A \mid \widetilde{\mathscr{H}}_{k}, k=1,2$, относительно разложения $\mathscr{H}=\widetilde{\mathscr{H}}_{1} \oplus \widetilde{\mathscr{H}}_{2} ;$ кроме того, если $P_{1}-$ проектор, осуществляющий разложсние $\mathscr{H}=\mathscr{H}_{1} \oplus \mathscr{H}_{2}$ (т.е. $\mathscr{H}_{1}=\operatorname{Im} P_{1}-$ образ проектора $P_{1}, \mathscr{H}_{2}=\operatorname{Im} P_{2}$, $P_{2}=I-P_{1}$ - дополнительный $\kappa P_{1}$ проектор), то проектор $\widetilde{P}_{1}$, осуществляющий разложение $\mathscr{H}=\widetilde{\mathscr{H}}_{1} \oplus \widetilde{\mathscr{H}}_{2}$, определяется формулой

$$
\widetilde{P}_{1}=U P_{1} U^{-1}
$$

3) если $A_{2}$ - инфинитезимальный оператор сильно непрерывной полугруппь операторов $T_{2}: \mathbb{R}_{+} \rightarrow$ End $\mathscr{H}_{2}$, то оператор $A_{1}$ является инфинитезимальным оператором силъно непрерывной полугруппы операторов

$$
T_{1}: \mathbb{R}_{+} \rightarrow \text { End } \mathscr{H}_{1}, \quad T_{1}(t)=U T_{2}(t) U^{-1}, \quad t \geqslant 0
$$

Следующий результат (см. [34]--[37]) является основным при использовании метода подобных операторов в вопросах получения оценок параметров экспоненциальной дихотомии и функции Грина возмущенного самосопряженного оператора $A$ с перечисленными выше свойствами.

Tеорема 22. Пусть $B \in$ End $\mathscr{H}$ и натуральное число $m$ въбрано так, что выполнено условие

$$
4 \gamma_{m}\|B\|=2 \pi \max \left\{\alpha_{m}^{-1}, \beta_{m}^{-1}\right\}\|B\|<1 .
$$

Тогда оператор $A-B$ подобен оператору $A-J_{m} X_{*}$, где оператор $X_{*} \in$ End $\mathscr{H}$ является решением (нелинейного) уравнения

$$
X=B \Gamma_{m} X-\left(\Gamma_{m} X\right)\left(J_{m} B\right)-\left(\Gamma_{m} X\right) J_{m}\left(B \Gamma_{m} X\right)+B=\Phi_{m}(X) .
$$

Данное решение можно найти методом простых итерачий, полагая $X_{0}=0$, $X_{1}=B, \ldots$ (оператор $\Phi_{m}:$ End $\mathscr{H} \rightarrow$ End $\mathscr{H}$ является сжимающим в шаpe $\{X \in$ End $\mathscr{H}:\|X-B\| \leqslant 3\|B\|\}$, и оператор $X_{*}$ лежит в этом шаре). Преобразование подобия оператора $A-B$ в оператор $A-J_{m} X_{*}$ осуществляет onepamop $I+\Gamma_{m} X_{*} \in$ End $\mathscr{H}$, m.e.

$$
(A-B)\left(I+\Gamma_{m} X_{*}\right)=\left(I+\Gamma_{m} X_{*}\right)\left(A-J_{m} X_{*}\right),
$$

при этом $\left\|\Gamma_{m} X_{*}\right\|<1$.

Из теоремы 18 и леммы 2 следует

Теорема 23. Пусть $B \in$ End $\mathscr{H}$ и натуралъное число $m$ удовлетворяет условию (6.1). Оператор $A-B$ и подобный ему (в силу теоремы 18) оператор $A(m)=A-J_{m} X_{*}$ являются инфинитезимальными операторами аналитических полугрупп операторов $T_{A-B}, T_{A(m)}: \mathbb{R}_{+} \rightarrow$ End $\mathscr{H}$ соответственно, причем верны равенства

$$
T_{A-B}(t)=\left(I+\Gamma_{m} X_{*}\right)^{-1} T_{A(m)}(t)\left(I+\Gamma_{m} X_{*}\right), \quad t \geqslant 0,
$$


где $X_{*}$ - решение нелинейного уравнения (6.2), удовлетворяющего условию $\left\|X_{*}-B\right\| \leqslant 3\|B\|$. Если полугруппа операторов $T_{A(m)}$ гиперболическал, то гиперболической будет полугруппа операторов $T_{A-B}$ и функиии Грина $G_{A(m)}$, $G_{A-B}: \mathbb{R} \rightarrow$ End $\mathscr{H}$, по ним построенные, связаны соотношением подобия

$$
G_{A-B}(t)=\left(I+\Gamma_{m} X_{*}\right)^{-1} G_{A(m)}(t)\left(I+\Gamma_{m} X_{*}\right), \quad t \in \mathbb{R},
$$

причем

$$
\left\|G_{A-B}(t)\right\| \leqslant \frac{1+4 \gamma_{m}\|B\|}{1-4 \gamma_{m}\|B\|}\left\|G_{A(m)}(t)\right\|, \quad t \in \mathbb{R} .
$$

Таким образом, метод подобных операторов позволяет благодаря равенствам $(6.3),(6.4)$ свести изучение полугруппы операторов $T_{A-B}$ к изучению полугруппы операторов $T_{A(m)}$ и получать оценки функции Грина $G_{A-B}$, используя оценки функции Грина $G_{A(m)}$ при условии гиперболичности полугруппы операторов $T_{A(m)}$.

Из вида оператора $A-J_{m} X_{*}$ следует, что он перестановочен со всеми проекторами $P_{(m)}=\sum_{k=1}^{m} P_{k}, P_{j}, j \geqslant m+1$. Следовательно, подпространства $\mathscr{H}_{(m)}=\operatorname{Im} P_{(m)}, \mathscr{H}_{j}=\operatorname{Im} P_{j}, j \geqslant m+1$, являются инвариантными относительно оператора $A-J_{m} X_{*}$. Таким же свойством инвариантности эти подпространства обладают и для операторов $T_{A(m)}(t), t \geqslant 0$.

Гильбертово пространство $\mathscr{H}$ представимо в виде

$$
\mathscr{H}=\mathscr{H}_{(m)} \oplus \mathscr{H}^{(m)}, \quad \mathscr{H}^{(m)}=\bigoplus_{k=m+1}^{\infty} \mathscr{H}_{k}
$$

ортогональной прямой суммы подпространств $\mathscr{H}_{(m)}, \mathscr{H}_{(k)}, k \geqslant m+1$. Поэтому полугруппа операторов $T_{A(m)}$ и функция Грина $G_{A(m)}$ (при условии гиперболичности полугруппы $\left.T_{A(m)}\right)$ допускают разложения вида

$$
\begin{gathered}
T_{A(m)}(t)=e^{A_{(m)} t} \oplus e^{A_{m+1} t} \oplus e^{A_{m+2} t} \oplus \cdots, \\
G_{A(m)}(t)=G_{(m)}(t) \oplus G_{A_{m+1}}(t) \oplus G_{A_{m+2}}(t) \oplus \cdots,
\end{gathered}
$$

где $A_{(m)}=A(m)\left|\mathscr{H}_{(m)}, A_{m+1}=A(m)\right| \mathscr{H}_{m+1}, A_{m+2}=A(m) \mid \mathscr{H}_{m+2}, \ldots$, функция Грина $G_{(m)}$ построена по оператору $A_{(m)}, G_{A_{m+1}}$ строится по оператору $A_{m+1}$ и т.д. Отметим, что операторы $A_{(m)}, A_{m+1}, \ldots$ действуют в конечномерных пространствах $\mathscr{H}_{(m)}, \mathscr{H}_{m+1}, \ldots$ соответственно, при этом из теоремы 18 (из равенства (6.2)) следует, что эти операторы определяются равенствами

$$
\begin{gathered}
A_{(m)}=P_{(m)} A P_{(m)}-P_{m} X_{*} P_{m}: \mathscr{H}_{(m)} \rightarrow \mathscr{H}_{(m)}, \\
A_{k}=P_{k} A P_{k}-P_{k} X_{*} P_{k}=\lambda_{k} P_{k}-P_{k} B P_{k}-P_{k}\left(X_{*}-B\right) P_{k} \in \text { End } \mathscr{H}_{k}, \\
k \geqslant m+1 .
\end{gathered}
$$

Из уравнения (6.2), решением которого является оператор $X_{*}$, и определения трансформаторов $\Gamma_{m}, J_{m}$ следуют равенства

$$
P_{k}\left(X_{*}-B\right) P_{k}=P_{k} B \Gamma_{m}\left(X_{*} P_{k}\right), \quad k \geqslant m+1 .
$$


Поскольку (используются равенства $\Gamma_{m}\left(P_{j} X_{*} P_{k}\right)=\left(\lambda_{j}-\lambda_{k}\right)^{-1} P_{j} X_{*} P_{k}, j \geqslant$ $m+1, j \neq k)$

$$
\begin{aligned}
& \left\|\Gamma_{m}\left(X_{*} P_{k}\right) x\right\|^{2}=\left\|\sum_{j \geqslant m+1, j \neq k} \frac{1}{\lambda_{j}-\lambda_{k}} P_{j} X_{*} P_{k} x\right\|^{2} \\
& =\sum_{j \geqslant m+1, j \neq k} \frac{1}{\left(\lambda_{j}-\lambda_{k}\right)^{2}}\left\|X_{*} P_{k} x\right\|^{2} \leqslant\left(\sum_{j \geqslant m+1, j \neq k} \frac{1}{\left(\lambda_{j}-\lambda_{k}\right)^{2}}\left\|P_{k} x\right\|^{2}\right)\|X\|_{*} \\
& \leqslant \frac{1}{\inf _{j \geqslant m+1, j \neq k}\left|\lambda_{k}-\lambda_{j}\right|^{2}}\left\|X_{*}\right\|^{2}\|x\|^{2}=\frac{1}{d_{k, m}}\left\|X_{*}\right\|^{2}\|x\|^{2}, \quad x \in \mathscr{H},
\end{aligned}
$$

то $\left\|\Gamma_{m}\left(X_{*} P_{k}\right)\right\| \leqslant d_{k}^{-1}\left\|X_{*}\right\|, k \geqslant m+1$, и поэтому

$$
\left\|P_{k}\left(X_{*}-B\right) P_{k}\right\| \leqslant d_{k, m}^{-1}\|B\|\left\|X_{*}\right\| \leqslant 4 d_{k, m}^{-1}\|B\|^{2}, \quad k \geqslant m+1 .
$$

Из представления (6.7) функции Грина $G_{A(m)}$ оператора $A(m)$ следует, что

$$
\begin{aligned}
\left\|G_{A(m)}(t) x\right\|^{2} & =\left\|G_{(m)}(t) P_{(m)} x\right\|^{2}+\sum_{k \geqslant m+1}\left\|G_{k}(t) P_{k} x\right\|^{2} \\
& \leqslant\left(\sup _{k \geqslant m+1}\left\{\left\|G_{(m)}(t)\right\|^{2},\left\|G_{k}(t)\right\|^{2}\right\}\right)\|x\|^{2}, \quad x \in \mathscr{H} .
\end{aligned}
$$

Поэтому имеют место оценки (см. (6.5))

$$
\begin{aligned}
\left\|G_{A-B}(t)\right\| & \leqslant \frac{1+4 \gamma_{m}\|B\|}{1-4 \gamma_{m}\|B\|}\left\|G_{A(m)}(t)\right\| \leqslant\left\|G_{A-B}(t)\right\| \\
& \leqslant \frac{1+4 \gamma_{m}\|B\|}{1-4 \gamma_{m}\|B\|} \sup _{k \geqslant m+1}\left\{\left\|G_{(m)}(t)\right\|,\left\|G_{k}(t)\right\|\right\}, \quad t \in \mathbb{R} .
\end{aligned}
$$

Таким образом, благодаря методу подобных операторов удается "расщепить" оператор $A-B$ и свести оценку функции Грина $G_{A-B}$ к оценке функций Грина $G_{(m)}, G_{k}, k \geqslant m+1$, которые строятся по операторам, действующим в конечномерных пространствах. Из представления (6.8) операторов $A_{k}, k \geqslant m+1$, следует, что

$$
e^{A_{k} t}=e^{\lambda_{k} t} e^{B_{k} t}, \quad t \in \mathbb{R}, \quad k \geqslant m+1,
$$

где $B_{k}=P_{k} B P_{k}-C_{k} \in$ End $\mathscr{H}_{k}, C_{k}=P_{k}\left(X_{*}-B\right) P_{k}, k \geqslant m+1$, причем из оценки (6.9) следует, что $\left\|C_{k}\right\| \leqslant 4 d_{k}^{-1}\|B\|^{2}$. Следовательно $\left\|B_{k}\right\| \leqslant\|B\|+$ $4 d_{k}^{-1}\|B\|^{2}$. Поэтому существует натуральное число $\ell=\ell(m) \geqslant m+1$ такое, что

$$
\left|\lambda_{k}\right|>\|B\|+4 d_{k}^{-1}\|B\|^{2}, \quad \lambda_{k}<0, \quad k \geqslant \ell .
$$

Из этих оценок и представления (6.6) следует, что операторная экспонента $t \mapsto e^{A_{k} t}: \mathbb{R}_{+} \rightarrow$ End $\mathscr{H}_{k}$ экспоненциально убывает и, следовательно, $G_{k}(t)=$ $e^{A_{k} t} ; G_{k}(t)=0, t<0, k \geqslant \ell$. Поэтому вопрос о гиперболичности полугруппы операторов $T_{A-B}$ свелся к вопросу о гиперболичности групп операторов $e^{A_{(m)} t}, e^{A_{k} t}, m+1 \leqslant k<\ell$. Итак, установлена 
Tеорема 24. Полугруппа операторов $T_{A-B}: \mathbb{R}_{+} \rightarrow$ End $\mathscr{H}$ гиперболична тогда и только тогда, когда выполнены следующие условия:

$$
\sigma(A(m)) \cap(i \mathbb{R})=\varnothing, \quad \sigma\left(A_{k}\right) \cap(i \mathbb{R})=\varnothing, \quad m+1 \leqslant k<\ell,
$$

где число $\ell$ таково, что имеет место (6.11). При выполнении этих условий функция Грина $G_{A-B}$ допускает оценки (6.10).

Отметим, что если число $m$ выбрано достаточно большим (удовлетворяющим наряду с условием (6.1) также оценке (6.11), где $\ell=m)$, то гиперболичность полугруппы $T_{A-B}$ эквивалентна выполнению условия $\sigma(A(m)) \cap(i \mathbb{R})=\varnothing$. Важно отметить также, что оператор $A(m)$ представим в виде

$$
A(m)=A^{0}(m)-X_{*}(m) \in \operatorname{End} \mathscr{H}_{(m)},
$$

где

$A^{0}(m)=P_{(m)} A P_{(m)}-P_{(m)} B P_{(m)}: \mathscr{H}_{(m)} \rightarrow \mathscr{H}_{(m)}, \quad X_{*}(m)=P_{(m)}\left(X_{*}-B\right) P_{(m)}$.

При этом $\lim _{m \rightarrow \infty}\left\|X_{*}(m)\right\|=0$.

Пусть $\mathscr{H}=L^{2}[0,1]$ - гильбертово пространство суммируемых с квадратом модуля комплекснозначных функций, определенных на промежутке $[0,1]$ и со скалярным произведением $(x, y)=\int_{0}^{1} x(t) \overline{y(t)} d t, x, y \in \mathscr{H}$. В качестве примера оператора, удовлетворяющего перечисленным выше условиям, является дифференциальный оператор

$$
A-B=\frac{d^{2}}{d t^{2}}-b: D(A-B)=D(A) \subset \mathscr{H} \rightarrow \mathscr{H},
$$

где $A=\frac{d^{2}}{d t^{2}}, B \in$ End $\mathscr{H},-$ оператор умножения на существенно ограниченную функцию $b:[0,1] \rightarrow \mathbb{C}$ (далее $\left.\|b\|_{\infty}=\operatorname{vrai}_{\sup _{t \in[0,1]}}|b(t)|\right)$ и $D(A)=\{x \in \mathscr{H}:$ $x$ абсолютно непрерывна и $\left.\dot{x} \in L^{2}[0,1]\right\}$.

Оператор $A$ является самосопряженным оператором с компактной резольвентой. Его спектр совпадает с множеством $\left\{-(\pi n)^{2}, n \geqslant 1\right\}$, собственные функции $e_{n}(t)=\sqrt{2} \sin \pi n t, t \in[0,1], n \geqslant 1$, образуют ортонормированный базис в $\mathscr{H}=L^{2}[0,1]$, причем $A e_{n}=-(\pi n)^{2} e_{n}, n \geqslant 1$. Оператор $B$ ограничен и $\|B\|=\|b\|_{\infty}$. Проектор Рисса $P_{n}$, построенный по одноточечному множеству $\left\{-\pi^{2} n^{2}\right\}$ имеет вид $P_{n} x=\left(x, e_{n}\right) e_{n}, n \geqslant 1$, причем $A P_{n}=-(\pi n)^{2} P_{n}$. В данном случае используемые в теореме 20 величины имеют вид

$$
d_{k}=\pi^{2}(2 k-1), \quad k \geqslant 2, \quad d_{1}=3 \pi^{2} ; \quad d_{k, m} \geqslant 2 m+3 .
$$

\section{§ 7. Комментарии к полученным результатам и некоторые дополнительные утверждения}

Для оценки параметров экспоненциальной устойчивости сильно непрерывной полугруппы операторов $T: \mathbb{R}_{+} \rightarrow$ End $\mathscr{H}$ можно использовать теорему Хилле-Филлипса-Иосида (см. [1]). Имеет место 
Теорема 25. Для того чтобъ числа $(M, w)$, где $M \geqslant 1, w<0$, были параметрами экспоненциальной устойчивости полугруппы $T$ с инфинитезимальньм оператором $A$, необходимо и достаточно, чтобы для всех $\lambda \in \mathbb{C}_{w}=$ $\{\mu \in \mathbb{C}: \operatorname{Re} \mu>w\}$ и $n \in \mathbb{N}$ имели место оценки

$$
\left\|R(\lambda, A)^{n}\right\| \leqslant M(\operatorname{Re} \lambda-w)^{-n}, \quad n \geqslant 1, \quad \operatorname{Re} \lambda>w .
$$

Условие (7.1) является труднопроверяемым из-за необходимости оценок норм степеней операторов $R(\lambda, A), \lambda \in \mathbb{C}_{w}$.

Если $\mathscr{H}$ - конечномерное пространство и оператор $A \in$ End $\mathscr{H}$ имеет спектр $\sigma(A)=\left\{\lambda_{1}, \ldots, \lambda_{m}\right\}$ с $\operatorname{Re} \lambda_{i}<0,1 \leqslant i \leqslant m$, то экспоненциальная устойчивость операторнозначной функции $t \mapsto e^{A t}: \mathbb{R}_{+} \rightarrow$ End $\mathscr{H}$ следует из следующей оценки Гельфанда-Шилова (см. [41; гл. II, §6] и [40; гл. I, упражнение 16]):

$$
\left\|e^{A t}\right\| \leqslant\left(\sum_{k=0}^{n-1} \frac{1}{k !}(2 t\|A\|)^{k}\right) e^{æ t}, \quad t \in \mathbb{R},
$$

где $æ=\max _{1 \leqslant i \leqslant m} \operatorname{Re} \lambda_{i}, n=\operatorname{dim} \mathscr{H}$. Эта оценка может быть использована для оценок параметров экспоненциальной устойчивости полугруппы операторов $T_{A-B}$ из $\S 6$. А именно, после преобразования подобия оценка функции Грина $G_{A-B}$ была сведена к оценке функции Грина $G_{A(m)}$, построенной по оператору $A(m)$. Оценка функции Грина при условии $\sigma(A(m)) \subset \mathbb{C}_{-}=\{\lambda \in \mathbb{C}$ : $\operatorname{Re} \lambda<0\}$ позволяет получить, используя оценки (7.2), достаточное условие экспоненциального убывания полугруппы операторов $T_{A-B}$ и соответствующие оценки параметров экспоненциальной устойчивости.

Экспоненциальное убывание полугруппы операторов $T: \mathbb{R}_{+} \rightarrow$ End $\mathscr{H}$, удовлетворяющей условию $\int_{0}^{\infty}\|T(t) x\|^{2} d t<\infty, x \in \mathscr{H}$, было установлено А. Пэзи (см. [42], [43]). Из принципа равномерной ограниченности для операторов (см. [1]) следует конечность величины

$$
\nu(T)=\sup _{\|x\| \leqslant 1} \int_{0}^{\infty}\|T(t) x\|^{2} d t .
$$

Поскольку полугруппа $T$ экспоненциально убывает, то $G_{A}(t)=T(t), t \geqslant 0$, и поэтому из равенства (7.3) следует, что $\nu(T)=\nu(A)$. Следовательно, применима теорема 7 для оценки такой полугруппы операторов. Итак, имеет место

Теорема 26. Пусть полугруппа операторов $T: \mathbb{R}_{+} \rightarrow$ End $\mathscr{H}$, удовлетворяет условию Пэзи (конечна величина (7.3)). Тогда T - экспоненииально устойчивая полугруппа операторов и для нее верна оценка

$$
\|T(t)\| \leqslant \sqrt{\nu(T)\left\|W^{-1}\right\|} \exp \left\{-\frac{1}{2 \nu(T)} t\right\}, \quad t \geqslant 0,
$$

при условии непрерывной обратимости решения $W \in$ End $\mathscr{H}$ уравнения Ляпунова (1.23), определяемого формулой (1.24). 
При условии, что оператор $A: D(A) \subset \mathscr{H} \rightarrow \mathscr{H}$ представим в виде $A=$ $A_{0}+B$, где $A_{0}^{*}=-A_{0}, B \in$ End $\mathscr{H}$, он является инфинитезимальным оператором (см. [1]) сильно непрерывной группы операторов $T: \mathbb{R}_{+} \rightarrow$ End $\mathscr{H}$ с числовой областью, для которой $\left|\beta_{\min }(A)\right| \leqslant\|B\|, \beta_{\max }(A) \leqslant\|B\|$. Поэтому выполнено предположение 1 из $\S 2$ и, следовательно, для такого оператора верно утверждение теоремы 6, в которой (в оценках (1.21) и (1.22)) величины $\left|\beta_{\min }(A)\right|, \beta_{\max }(A)$ могут быть заменены на число $\|B\|$.

Комментируя результаты $\S 6$, отметим, что если линейное отношение $\mathscr{A} \in$ $L R C(\mathscr{X})$ является линейным замкнутым оператором, то в статье [30] было установлено, что оператор $\mathscr{A} \in L O\left(\ell^{p}\right)$ обратим одновременно во всех пространствах $\ell^{p}=\ell^{p}(\mathbb{Z}, \mathscr{X}), p \in[1, \infty]$, и получены следующие оценки для функции Грина $G: \mathbb{Z} \rightarrow$ End $\mathscr{X}$ вида

$$
\begin{gathered}
\|G(k)\| \leqslant\left\|\mathscr{A}^{-1}\right\|, \quad k \in \mathbb{Z}, \\
\|G(k)\| \leqslant 2\left\|\mathscr{A}^{-1}\right\|\left(1+\frac{1}{2\left\|\mathscr{A}^{-1}\right\|}\right)^{1-k}, \quad k \geqslant 1, \\
\|G(k)\| \leqslant 2\left\|\mathscr{A}^{-1}\right\|\left(1-\frac{1}{2\left\|\mathscr{A}^{-1}\right\|}\right)^{1-k},
\end{gathered}
$$

если $\left\|\mathscr{A}^{-1}\right\|>1, k \leqslant-1$, и $G(k)=0$, если $\left\|\mathscr{A}^{-1}\right\| \leqslant 1$ (если $\mathscr{X}$ - гильбертово пространство, то во всех этих оценках величина $\left\|\mathscr{A}^{-1}\right\|$ может быть заменена на величину $\left.\gamma_{\mathbb{T}}(A)\right)$. Кроме того, верна оценка

$$
\left\|\mathscr{A}^{-1}\right\|_{q} \leqslant 8\left\|\mathscr{A}^{-1}\right\|_{p}^{2}-\left\|\mathscr{A}^{-1}\right\|_{p}+1,
$$

где символы $\left\|\mathscr{A}^{-1}\right\|_{q},\left\|\mathscr{A}^{-1}\right\|_{p}$ обозначают норму оператора $\mathscr{A}^{-1}$ в пространствах $\ell^{q}$ и $\ell^{p}$ соответственно. В частности, если $p=2$ и $\mathscr{X}-$ гильбертово пространство, то $\left\|\mathscr{A}^{-1}\right\|_{2}=\gamma_{\mathbb{T}}(A)$, и поэтому

$$
\left\|\mathscr{A}^{-1}\right\|_{q} \leqslant 8 \gamma_{\mathbb{T}}(A)^{2}-\gamma_{\mathbb{T}}(A)+1, \quad q \in[1, \infty] .
$$

Если оператор $A$ принадлежит алгебре End $\mathscr{X}$, то $\mathscr{A} \in$ End $\ell^{p}, p \in[1, \infty]$, и для оценки функции Грина $G: \mathbb{Z} \rightarrow$ End $\mathscr{X}$ можно использовать результаты статей [43]-[47] об оценках элементов обратных матриц для ограниченных операторов (см. также [48]). Так, из [43; теорема 3], в частности, следует оценка $\sum_{k \in \mathbb{Z}}\|G(k)\| \leqslant 16 æ(A) \gamma_{\mathbb{T}}(A)$, где $(A)=\max \{1,\|A\|\}$, в предположении, что $\mathscr{X}$ - гильбертово пространство.

В монографии [14; п. 8.5] для оператора $A \in \operatorname{End} \mathscr{X}, \operatorname{dim} \mathscr{X}<\infty$, для которого $r(A)<1$, получены оценки норм степеней оператора $A$ вида

$$
\left\|A^{k}\right\| \leqslant \sup _{|\lambda|=r(A)^{1 / 2}}\|R(\lambda, A)\|(r(A))^{(k+1) / 2}, \quad k \geqslant 2 .
$$

Такие оценки верны и для $A \in$ End $\mathscr{X}$ в случае произвольного банахова пространства $\mathscr{X}$, если $r(A)<1$.

Пусть $\operatorname{dim} \mathscr{X}=m<\infty$ и $A \in$ End $\mathscr{X}$ с $r(A)<1$. Для оценок величин $\left\|A^{n}\right\|$, $n \geqslant m$, можно использовать отмеченный нами метод Гельфанда-Шилова, который ими применялся для оценки (7.2) операторной экспоненты. Предположим вначале, что собственные значения оператора $A$ простые, и пусть $\sigma(A)=$ 
$\left\{\lambda_{1}, \ldots, \lambda_{m}\right\}$. Тогда согласно метода Гельфанда-Шилова, основанного на использовании интерполяционного многочлена в форме Ньютона, для любого $n \geqslant m$ оператор $A^{n}$ представим в виде

$$
A^{n}=a_{1} I+a_{2} A+\cdots+a_{m} A^{m-1},
$$

где числа $a_{k} \in \mathbb{C}, 1 \leqslant k \leqslant m$, допускают оценки вида

$$
\begin{gathered}
\left|a_{1}\right| \leqslant \max _{\lambda \in \mathscr{B}}\left|\lambda^{n}\right|=r(A)^{n}, \\
\left|a_{k+1}\right| \leqslant \frac{1}{k !} n(n-1) \cdots(n-k-1) \max _{\lambda \in \mathscr{B}}|\lambda|^{n-k}=C_{n}^{k} r(A)^{n-k}, \quad 1 \leqslant k \leqslant m-1 .
\end{gathered}
$$

Здесь символ $\mathscr{B}$ обозначает выпуклую оболочку в $\mathbb{C}$ собственных значений $\lambda_{1}, \ldots, \lambda_{m}$ оператора $A$. Если собственные значения не являются простыми, то следует рассмотреть последовательность операторов $\left(A_{k}\right)$ из End $\mathscr{X}$, сходящуюся к $A$ и в которой каждый оператор имеет простые собственные значения.

Из приведенных оценок следует

Теорема 27. Имеют место оценки

$$
\left\|A^{n}\right\| \leqslant \sum_{k=0}^{m-1} C_{n}^{k} r(A)^{n-k}, \quad n \geqslant m .
$$

М. Гилем в работе [49] разработан метод оценки норм степеней оператора $A \in \operatorname{End} \mathbb{C}^{m}$ (а также $e^{t A}$ ), использующий величину

$$
g(A)=\left(N^{2}(A)-\sum_{k=1}^{m}\left|\lambda_{k}(A)\right|^{2}\right)^{1 / 2} \leqslant \frac{1}{2} N^{2}\left(A^{*}-A\right),
$$

где $N(A)=\left(\operatorname{tr}\left(A A^{*}\right)\right)^{1 / 2}$ - норма Гильберта-Шмидта оператора $A$. А именно, имеют место оценки

$$
\left\|A^{n}\right\| \leqslant \sum_{k=0}^{m-1} \frac{\gamma_{m, k} n ! g^{k}(A) r(A)^{n-k}}{(n-k) ! k !}, \quad n \geqslant 1,
$$

где

$$
\gamma_{m, k}=\sqrt{\frac{C_{m-1}^{k}}{(m-1) !}}, \quad 1 \leqslant k \leqslant m-1, \quad \gamma_{m, 0}=1 .
$$

Отметим, что в статье [50; теоремы 3-5] получены оценки решений дифференциальных уравнений с периодическими коэффициентами, основываясь на использовании дифференциального матричного уравнения Ляпунова. В статье [51] приведено подробное доказательство теоремы 13.

\section{Список литературы}

[1] Э. Хилле, Р. Филлипс, Функиионалъный анализ и полугруппы, ИЛ, М., 1962, 829 с.; пер. с англ.: E. Hille, R. S. Phillips, Functional analysis and semi-groups, rev. ed., Amer. Math. Soc. Colloq. Publ., 31, Amer. Math. Soc., Providence, R. I., 1957, xii+808 pp. 
[2] А. Г. Баскаков, "Полугруппы разностных операторов в спектральном анализе линейных дифференциальных операторов", Функи. анализ и его прил., 30:3 (1996), 1-11; англ. пер.: A. G. Baskakov, "Semigroups of difference operators in spectral analysis of linear differential operators", Funct. Anal. Appl., 30:3 (1996), 149-157.

[3] C. Chicone, Yu. Latushkin, Evolution semigroups in dynamical systems and differential equations, Math. Surveys Monogr., 70, Amer. Math. Soc., Providence, RI, 1999, $\mathrm{x}+361 \mathrm{pp}$.

[4] А.Г. Баскаков, Ю.Н. Синтяев, "Разностные операторы в исследовании дифференциальных операторов: оценки решений”, Дифферени. уравнения, 46:2 (2010), 210-219; англ. пер.: A. G. Baskakov, Yu. N. Sintyaev, "Finite-difference operators in the study of differential operators: Solution estimates", Differ. Equ., 46:2 (2010), $214-223$.

[5] А. Г. Баскаков, "Исследование линейных дифференциальных уравнений методами спектральной теории разностных операторов и линейных отношений", $У М H$, 68:1(409) (2013), 77-128; англ. пер.: А. G. Baskakov, "Analysis of linear differential equations by methods of the spectral theory of difference operators and linear relations", Russian Math. Surveys, 68:1 (2013), 69-116.

[6] J. Prüss, "On the spectrum of $C_{0}$-semigroups", Trans. Amer. Math. Soc., 284:2 (1984), 847-857.

[7] K.-J. Engel, R. Nagel, One-parameter semigroups for linear evolution equations, Grad. Texts in Math., 194, Springer-Verlag, New York, 2000, xxii+586 pp.

[8] А. Г. Чшиев, "Теорема Герхарда-Прюсса для некоторого класса вырожденных полугрупп операторов", Матем. заметки, 94:3 (2013), 426-440; англ. пер.: A. G. Chshiev, "The Gearhart-Prüss theorem for a class of degenerate semigroups of operators", Math. Notes, 94:3 (2013), 400-413.

[9] А.Г. Баскаков, “Линейные отношения как генераторы полугрупп операторов", Матем. заметки, 84:2 (2008), 175-192; англ. пер.: A. G. Baskakov, "Linear relations as generators of semigroups of operators", Math. Notes, 84:2 (2008), 166-183.

[10] А.Г. Баскаков, А.А. Воробьев, М.Ю.Романова, "Гиперболические полугруппы операторов и уравнение Ляпунова", Матем. заметки, 89:2 (2011), 190-203; англ. пер.: A. G. Baskakov, A. A. Vorobjev, M. Yu. Romanova, "Hyperbolic operator semigroups and Lyapunov's equation", Math. Notes, 89:2 (2011), 194-205.

[11] А.Г. Баскаков, К. С. Кобычев, “Оценки оператора вложения пространства Соболева периодических функций и оценки решений дифференциальных уравнений с периодическими коэффициентами", Дифферени. уравнения, 47:5 (2011), 611-620; англ. пер.: A. G. Baskakov, K. S. Kobychev, "Estimates for the embedding operator of a Sobolev space of periodic functions and for the solutions of differential equations with periodic coefficients", Differ. Equ., 47:5 (2011), 609-619.

[12] Л. А. Люстерник, В.И. Соболев, Элементы функиионалъного анализа, 2-е изд., Наука, М., 1965, 520 с.; англ. пер.: L. A. Lusternik, V. J. Sobolev, Elements of functional analysis, Hindustan Publishing Corp., Delhi; John Wiley \& Sons, Inc., New York, 1974, x+322 pp.

[13] Т. Като, Теория возмущений линейнъх операторов, Мир, М., 1972, 740 с.; пер. с англ.: Т. Kato, Perturbation theory for linear operators, Grundlehren Math. Wiss., 132, Springer-Verlag, New York, 1966, xix+592 pp.

[14] С.К. Годунов, Современные аспекты линейной алгебры, Научн. кн., Новосибирск, 1997, 390 с.; англ. пер.: S. K. Godunov, Modern aspects of linear algebra, Transl. Math. Monogr., 175, Amer. Math. Soc., Providence, RI, 1998, xvi+303 pp.

[15] А.Г. Баскаков, "Оценки ограниченных решений линейных дифференциальных уравнений", Дифферени. уравнения, 39:3 (2003), 413-415; англ. пер.: 
A. G. Baskakov, "Estimates of bounded solutions of linear differential equations", Differ. Equ., 39:3 (2003), 447-450.

[16] А. И. Перов, “Частотные признаки существования ограниченных решений”, Дифферени. уравнения, 43:7 (2007), 896-904; англ. пер.: А. I. Perov, "Frequency tests for the existence of boundary solutions", Differ. Equ., 43:7 (2007), 916-924.

[17] С. Г. Крейн, Линейные дифференииальные уравнения в банаховом пространстве, Наука, М., 1967, 464 с.; англ. пер.: S. G. Kreйn, Linear differential equations in Banach space, Transl. Math. Monogr., 29, Amer. Math. Soc., Providence, R.I., 1971, $\mathrm{v}+390 \mathrm{pp}$.

[18] С. К. Годунов, Ю. М. Нечепуренко, “Оценки для главной и жесткой компонент на основе интегрального критерия качества дихотомии", Журн. въчисл. матем. и матем. физ., 40:1 (2000), 35-42; англ. пер.: S. K. Godunov, Yu. M. Nechepurenko, "Bounds for the principal and stiff components based on the integral performance criterion for dichotomy", Comput. Math. Math. Phys., 40:1 (2000), 32-39.

[19] Ю.М. Нечепуренко, "Об оценке нормы матричной экспоненты", Докл. РАН, 377:5 (2001), 597-600; англ. пер.: Yu. M. Nechepurenko, "On a bound for the norm of a matrix exponential", Dokl. Math., 63:2 (2001), 233-236.

[20] Ю. М. Нечепуренко, "Новая оценка нормы матрицы Грина", Докл. РАН, 378:4 (2001), 450-451; англ. пер.: Yu. M. Nechepurenko, "A new bound for the norm of the Green matrix", Dokl. Math., 63:3 (2001), 362-363.

[21] Ю. М. Нечепуренко, "Оценка нормы матричной экспоненты через норму решения уравнения Ляпунова и границы хаусдорфова множества”, Журн. вычисл. матем. и матем. физ., 42:2 (2002), 131-141; англ. пер.: Yu. M. Nechepurenko, "Bounds for the matrix exponential based on the Lyapunov equation and limits of the Hausdorff set", Comput. Math. Math. Phys., 42:2 (2002), 125-134.

[22] А. Я. Булгаков, "Обоснование гарантированной точности выделения инвариантных подпространств несамосопряженных матриц", Тр. Ин-та матем. СО АН CCCP, 15, Новосибирск, 1989, 12-93; англ. пер.: А. Ya. Bulgakov, "The basis of guaranteed accuracy in the problem of separation of invariant subspaces for nonselfadjoint matrices. I", Siberian Adv. Math., 1:1 (1991), 64-108.

[23] T. Eisner, Stability of operators and operator semigroups, Oper. Theory Adv. Appl., 209, Birkhäuser Verlag, Basel, 2010, viii+204 pp.

[24] A. Aldroubi, A. Baskakov, I. Krishtal, "Slanted matrices, Banach frame, and sampling", J. Funct. Anal., 255:7 (2008), 1667-1691.

[25] А.Г. Баскаков, К.И. Чернышов, “Спектральный анализ линейных отношений и вырожденные полугруппы операторов", Матем. сб., 193:11 (2002), 3-42; англ. пер.: A. G. Baskakov, K.I. Chernyshov, "Spectral analysis of linear relations and degenerate operator semigroups", Sb. Math., 193:11 (2002), 1573-1610.

[26] А. Г. Баскаков, "Спектральный анализ дифференциальных операторов с неограниченными операторными коэффициентами, разностные отношения и полугруппы разностных отношений", Изв. РАН. Сер. матем., 73:2 (2009), 3-68; англ. пер.: А.G. Baskakov, "Spectral analysis of differential operators with unbounded operator-valued coefficients, difference relations and semigroups of difference relations", Izv. Math., 73:2 (2009), 215-278.

[27] А. Г. Баскаков, “Теория представлений банаховых алгебр, абелевых групп и полугрупп в спектральном анализе линейных операторов", Функииональный анализ, СМФН, 9, МАИ, М., 2004, 3-151; англ. пер.: А. G. Baskakov, "Representation theory for Banach algebras, Abelian groups, and semigroups in the spectral analysis of linear operators", J. Math. Sci. (N. Y.), 137:4 (2006), 4885-5036.

[28] R. Cross, Multivalued linear operators, Monogr. Textbooks Pure Appl. Math., 213, Marcel Dekker, Inc., New York, 1998, x+335 pp. 
[29] A. Favini, A. Yagi, Degenerate differential equations in Banach spaces, Monogr. Textbooks Pure Appl. Math., 215, Marcel Dekker, Inc., New York, 1999, xii+313 pp.

[30] А.Г. Баскаков, А.И. Пастухов, "Спектральный анализ оператора взвешенного сдвига с неограниченными операторными коэффициентами", Сиб. матем. журн., 42:6 (2001), 1231-1243; англ. пер.: A. G. Baskakov, A. I. Pastukhov, "Spectral analysis of a weighted shift operator with unbounded operator coefficients", Siberian Math. J., 42:6 (2001), 1026-1035.

[31] М. С. Бичегкуев, "Об ограниченных решениях разностных включений", Изв. вузов. Матем., 8 (2008), 16-24; англ. пер.: M.S. Bichegkuev, "Bounded solutions of difference inclusions", Russian Math. (Iz. VUZ), 52:8 (2008), 12-19.

[32] А.Г. Баскаков, "Дихотомия спектра несамосопряженных операторов", Сиб. матем. журн., 32:3 (1991), 24-30; англ. пер.: A. G. Baskakov, "Dichotomy of the spectrum of non-self-adjoint operators", Siberian Math. J., 32:3 (1991), 370-375.

[33] А.Г. Баскаков, В.В.Юргелас, "Индефинитная диссипативность и обратимость линейных дифференциальных операторов”, Укр. матем. журн., 41:12 (1989), 1613-1618; англ. пер.: А. G. Baskakov, V.V. Yurgelas, "Indefinite dissipativity and invertibility of linear differential operators", Ukrainian Math. J., 41:12 (1989), $1385-1389$.

[34] А.Г. Баскаков, "Методы абстрактного гармонического анализа в теории возмущений линейных операторов", Сиб. матем. журн., 24:1 (1983), 21-39; англ. пер.: A. G. Baskakov, "Methods of abstract harmonic analysis in the perturbation of linear operators", Siberian Math. J., 24:1 (1983), 17-32.

[35] А. Г. Баскаков, "Теорема о расщеплении оператора и некоторые смежные вопросы аналитической теории возмущений”, Изв. АН СССР. Сер. матем., 50:3 (1986), 435-457; англ. пер.: A. G. Baskakov, "A theorem on splitting an operator, and some related questions in the analytic theory of perturbations", Math. USSR-Izv., 28:3 (1987), 421-444.

[36] А.Г. Баскаков, "Спектральный анализ возмущенных неквазианалитических и спектральных операторов", Изв. РАН. Сер. матем., 58:4 (1994), 3-32; англ. пер.: A. G. Baskakov, "Spectral analysis of perturbed nonquasianalytic and spectral operators", Russian Acad. Sci. Izv. Math., 45:1 (1995), 1-31.

[37] А. Г. Баскаков, А. В. Дербушев, А. О. Щербаков, "Метод подобных операторов в спектральном анализе несамосопряженного оператора Дирака с негладким потенциалом", Изв. РАН. Сер. матем., 75:3 (2011), 3-28; англ. пер.: А. G. Baskakov, A. V. Derbushev, A. O. Shcherbakov, "The method of similar operators in the spectral analysis of non-self-adjoint Dirac operators with non-smooth potentials", Izv. Math., 75:3 (2011), 445-469.

[38] А. М. Гомилко, "Об условиях на производящий оператор равномерно ограниченной $C_{0}$-полугруппы операторов", Функи. анализ и его приложения, 33:4 (1999), 66-69; англ. пер.: А. M. Gomilko, "Conditions on the generator of a uniformly bounded $C_{0}$-semigroup", Funct. Anal. Appl., 33:4 (1999), 294-296.

[39] Д. Хенри, Геометрическая теория полулинейных параболических уравнений, Мир, М., 1985, 376 с.; пер. с англ.: D. Henry, Geometric theory of semilinear parabolic equations, Lecture Notes in Math., 840, Springer-Verlag, Berlin-New York, 1981, iv +348 pp.

[40] Ю.Л. Далецкий, М.Г. Крейн, Устойчивость решений дифференииальных уравнений в банаховом пространстве, Наука, М., 1970, 534 с.; англ. пер.: Yu. L. Daletskii, M. G. Krein, Stability of solutions of differential equations in Banach space, Transl. Math. Monogr., 43, Amer. Math. Soc., Providence, RI, 1974, vi+386 pp.

[41] И. М. Гельфанд, Г.Е. Шилов, Некоторые вопросы теории дифференииальных уравнений, Обобщенные функции, Вып. 3, Физматлит, М., 1958, 274 с.; англ. пер.: I. M. Gel'fand, G. E. Shilov, Generalized functions, v. 3: Theory of differential equations, Academic Press, New York-London, 1967, x+222 pp. 
[42] A. Pazy, Semigroups of linear operators and applications to partial differential equations, Appl. Math. Sci., 44, Springer-Verlag, New York, 1983, viii+279 pp.

[43] А.Г. Баскаков, "Оценки элементов обратных матриц и спектральный анализ линейных операторов”, Изв. РАН. Сер. матем., 61:6 (1997), 3-26; англ. пер.: A. G. Baskakov, "Estimates for the entries of inverse matrices and the spectral analysis of linear operators", Izv. Math., 61:6 (1997), 1113-1135.

[44] А. Г. Баскаков, "Теорема Винера и асимптотические оценки элементов обратных матриц", Функи. анализ и его прил., 24:3 (1990), 64-65; англ. пер.: A. G. Baskakov, "Wiener's theorem and the asymptotic estimates of the elements of inverse matrices", Funct. Anal. Appl., 24:3 (1990), 222-224.

[45] А. Г. Баскаков, "Абстрактный гармонический анализ и асимптотические оценки элементов обратных матриц", Матем. заметки, 52:2 (1992), 17-26; англ. пер.: A. G. Baskakov, "Abstract harmonic analysis and asymptotic estimates of elements of inverse matrices", Math. Notes, 52:2 (1992), 764-771.

[46] А.Г. Баскаков, "Асимптотические оценки элементов матриц обратных операторов и гармонический анализ", Сиб. матем. журн., 38:1 (1997), 14-28; англ. пер.: A. G. Baskakov, "Asymptotic estimates for the entries of the matrices of inverse operators and harmonic analysis", Siberian Math. J., 38:1 (1997), 10-22.

[47] A. G. Baskakov, I. A. Krishtal, "Memory estimation of inverse operators", J. Funct Anal., 267:8 (2014), 2551-2605.

[48] A. G. Baskakov, I. A. Krishtal, "Spectral analysis of operators with the two-point Bohr spectrum", J. Math. Anal. Appl., 302:2 (2005), 420-439.

[49] M.I. Gil', "Difference equations in normed spaces. Stability and oscillations", North-Holland Math. Stud., 206, Elsevier, Amsterdam, 2007, xvi+362 pp.

[50] Г. В. Демиденко, И. И. Матвеева, "Об устойчивости решений линейных систем с периодическими коэффициентами”, Сиб. матем. журн., 42:2 (2001), 332-348; англ. пер.: G.V. Demidenko, I. I. Matveeva, "On stability of solutions to linear systems with periodic coefficients", Siberian Math. J., 42:2 (2001), 282-296.

[51] М. С. Бичегкуев, "К спектральному анализу разностных и дифференциальных операторов в весовых пространствах", Матем. сб., 204:11 (2013), 3-20; англ. пер.: M.S. Bichegkuev, "Spectral analysis of difference and differential operators in weighted spaces", Sb. Math., 204:11 (2013), 1549-1564.

\section{Анатолий Григорьевич Баскаков (Anatoliy G. Baskakov)}

